\title{
PLURI-POTENTIAL THEORY ON GRAUERT TUBES OF REAL ANALYTIC RIEMANNIAN MANIFOLDS, I
}

\begin{abstract}
Analogues of the some basic notions of pluri-potential theory on strictly pseudoconvex domains in $\mathbb{C}^{m}$ are developed for Grauert tubes $M_{\tau}$ in complexifications of real analytic Riemannian manifolds $(M, g)$. In particular, the normalized logarithm of the complexified spectral projector $\Pi_{I_{\lambda}}^{\mathbb{C}}(\zeta, \bar{\zeta})$ is the analogue of the Siciak-Zaharjuta extremal plurisubharmonic function. It is shown that $\frac{1}{\lambda} \log \Pi_{I_{\lambda}}^{\mathbb{C}}(\zeta, \bar{\zeta}) \rightarrow \sqrt{\rho}(\zeta)$, where $\sqrt{\rho}$ is the Grauert tube function. We give several applications to analytic continuations of eigenfunctions: to norm estimates, triple product integrals and to complex nodal sets.
\end{abstract}

In the study of eigenfunctions of the Laplacian $\Delta_{g}$ on a real analytic Riemannian manifold $(M, g)$ of dimension $m$, it is often useful to analytically continue an orthonormal basis $\left\{\varphi_{\lambda_{j}}\right\}$ of eigenfunctions,

$$
\Delta_{g} \varphi_{\lambda_{j}}=\lambda_{j}^{2} \varphi_{\lambda_{j}}, \quad\left\langle\varphi_{\lambda_{j}}, \varphi_{\lambda_{k}}\right\rangle=\delta_{j k}, \quad\left(\lambda_{0}=0<\lambda_{1} \leq \lambda_{2} \leq \cdots\right)
$$

into the complexification $M_{\mathbb{C}}$ of $M$. As recalled in $\$ 1$, eigenfunctions admit analytic continuations $\varphi_{\lambda_{j}}^{\mathbb{C}}$ to a maximal uniform 'Grauert tube'

$$
M_{\tau}=\left\{\zeta \in M_{\mathbb{C}}, \sqrt{\rho}(\zeta)<\tau\right\}
$$

independent of $\lambda_{j}$, where the radius is measured by the Grauert tube function $\sqrt{\rho}(\zeta)$ corresponding to $g$ (see §1: [LS1, GS1]). Analytic continuation of eigenfunctions and spectral projections (2)-(3) to Grauert tubes have applications to nodal geometry [DF, Lin, Z3, TZ, RZ], analytic wave front sets [Leb, GLS, tunnelling estimates [HS, Mar], Paley-Wiener theorems [G], invariant triple products [Sar, BR], random waves [Z2] and Agmon estimates for eigenfunctions in the classically forbidden region (see e.g. To]).

Grauert tubes are strictly pseudo-convex Stein manifolds, and in some ways are analogous to strictly pseudo-convex domains in $\mathbb{C}^{m}$ and to Hermitian unit bundles in negative line bundles. The purpose of this article and its sequel [Z1] is to extend to Grauert tubes some of the basic notions and results of PSH (pluri-subharmonic) function theory on stricty pseudo-convex domains in $\mathbb{C}^{n}$ (cf. $[\mathrm{K}, \mathrm{BL}$ ), and their recent generalization of this theory to Kähler manifolds in [GZ]. The basic theme is to use analytic continuations of eigenfunctions $\left\{\varphi_{\lambda_{j}}^{\mathbb{C}}\right\}$ in place of holomorphic polynomials of degree $\sim \lambda_{j}$ on $\mathbb{C}^{m}$ or holomorphic sections of line bundles of degree $\sim \lambda_{j}$ over a Kähler manifold. The primary objects are the analytic continuations of the spectral projections kernels of $\Delta_{g}$,

$$
\Pi_{I_{\lambda}}^{\mathbb{C}}(\zeta, \bar{\zeta})=\sum_{j: \lambda_{j} \in I_{\lambda}}\left|\varphi_{\lambda_{j}}^{\mathbb{C}}(\zeta)\right|^{2}
$$

which are of exponential growth, and their 'tempered' analogues,

$$
P_{I_{\lambda}}^{\tau}(\zeta, \bar{\zeta})=\sum_{j: \lambda_{j} \in I_{\lambda}} e^{-\tau \lambda_{j}}\left|\varphi_{\lambda_{j}}^{\mathbb{C}}(\zeta)\right|^{2}, \quad(\sqrt{\rho}(\zeta) \leq \tau),
$$

Date: October 15, 2018.

Research partially supported by NSF grant \# DMS-0904252. 
where $I_{\lambda}$ could be a short interval $[\lambda, \lambda+1]$ of frequencies or a long window $[0, \lambda]$. In this article, we only consider long windows $I_{\lambda}=[0, \lambda]$ while in [Z1] we refine the results to short windows using the long time behavior of the complexified geodesic flow. The tempered kernels $P_{I_{\lambda}}^{\tau}(\zeta, \bar{\zeta})$ are in some ways analogous to the 'density of states function' or Bergman kernel on the diagonal in the setting of positive line bundles over Kähler manifolds [Z4]. We gave some initial results on these kernels in [Z2], by somewhat different methods.

A basic notion in PSH theory is that of maximal PSH functions satisfying bounds and the (non-obviously) equivalent Siciak-Zaharjuta extremal PSH functions. We define a Grauert tube analogue of the Siciak-Zaharjuta extremal function and show in Theorem 1 that it is the same as the Grauert tube function. The proof is to relate both to the complexified spectral projections (2) which are defined in terms of eigenfunctions. The proof only requires a one term local Weyl law (see Theorem 2), which also gives improvements on the pointwise bounds on complexified eigenfunctions in GLS. The result can be improved to a rather interesting two term Weyl law of Safarov-Vasilliev type [SV]; this is carried out in the sequel [Z1].

This article also contains a general type of result on integrals of triple products of eigenfunctions (Proposition 11). The precise results depend on the radius of the maximal Grauert tube. We point out that there are two possible definitions (see Definition 1.1), an analytic maximal radius and a geometric maximal radius; in $\$ 11.1$ we sketch a proof that these radii are the same. Finally, we build on [AS] to give a result on limit distribution of complex zeros of eigenfunctions on locally symmetric manifolds of non-positive curvature.

In keeping with the nature of this symposium, this article is partly expository. In particular, we review the construction of the Hadamard parametrix of the wave kernel and its holomorphic extension to Grauert tubes. We also illustrate the issues and notions with examples from surfaces of constant curvature. We thank J. Sjöstrand and the referee for helpful discussions/comments on the material.

0.1. A Siciak-Zaharjuta extremal function for Grauert tubes. Before defining the analogues, let us first recall the definitions of relative maximal or extremal PSH functions satisfying bounds on a pair $E \subset \Omega \subset \mathbb{C}^{m}$ where $\Omega$ is a bounded open set. There are two definitions:

- The pluri-complex Green's function relative to a subset $E \subset \Omega$, defined [Br, Sic] as the upper semi-continuous regularization $V_{E, \Omega}^{*}$ of

$$
V_{E, \Omega}(z)=\sup \left\{u(z): u \in P S H(\Omega),\left.u\right|_{E} \leq 0,\left.u\right|_{\partial \Omega} \leq 1\right\} .
$$

- The Siciak-Zaharjuta extremal function relative to $E \subset \Omega$, defined by

$$
\log \Phi_{E}^{N}(\zeta)=\sup \left\{\frac{1}{N} \log \left|p_{N}(\zeta)\right|: p \in \mathcal{P}_{E}^{N}\right\}, \quad \log \Phi_{E}=\limsup _{N \rightarrow \infty} \log \Phi_{E}^{N},
$$

where $\mathcal{P}_{E}^{N}=\left\{p \in \mathcal{P}^{N}:\|p\|_{E} \leq 1,\|p\|_{\Omega} \leq e^{N}\right\}$.

Here, $\|f\|_{E}=\sup _{z \in E}|f(z)|$ and $\mathcal{P}^{N}$ denotes the space of all complex analytic polynomials of degree $N$. Siciak proved that $\log \Phi_{E}=V_{E}$ (see [Sic2] Theorem 1, and [K], Theorem 5.1.7). Intuitively, there are enough polynomials that one can obtain the sup by restricting to polynomials. 
There are analogous definitions in the case of unit co-disc bundles in the dual of a positive holomorphic Hermitian line bundle $L \rightarrow M$ over a Kähler manifold. In the case of $\mathbb{C P}^{n}$, one defines

$$
V_{K}(z)=\sup \{u(z): u \in \mathcal{L}, u \leq 0 \text { on } K\}
$$

where $\mathcal{L}$ denotes the Lelong class of all global plurisubharmonic (PSH) functions $u$ on $\mathbb{C}^{n}$ with $u(z) \leq c_{u}+\log (1+|z|)$. We refer to [GZ for further information in the Kähler setting.

We now define an analogue of the Siciak-Zaharjuta extremal function for Grauert tubes in the special case where $E=M$, the underlying real manifold. A generalization to other sets $E \subset M_{\tau}$ is discussed in $§ 10.3$. The Riemannian analogue of $\mathcal{P}^{N}$ is the space

$$
\mathcal{H}^{\lambda}=\left\{p=\sum_{j: \lambda_{j} \in I_{\lambda}} a_{j} \varphi_{\lambda_{j}}^{\mathbb{C}}, a_{1}, \ldots, a_{N(\lambda)} \in \mathbb{R}\right\}
$$

spanned by the eigenfunctions with 'degree' $\lambda_{j} \leq \lambda$. Here, $N(\lambda)=\#\left\{j: \lambda_{j} \in I_{\lambda}\right\}$. As above, we could let $I_{\lambda}=[0, \lambda]$ or $I_{\lambda}=[\lambda, \lambda+c]$ for some $c>0$. It is simpler to work with $L^{2}$ based norms than sup norms, and so we define

$$
S \mathcal{H}_{M}^{\lambda}=\left\{\psi=\sum_{j: \lambda_{j} \leq \lambda} a_{j} \varphi_{\lambda_{j}}^{\mathbb{C}}, \quad \sum_{j=1}^{N(\lambda)}\left|a_{j}\right|^{2}=1\right\} .
$$

Definition 1. The Riemannian Siciak-Zaharjuta extremal function (with respect to the real locus $M)$ is defined by:

$$
\left\{\begin{array}{l}
\log \Phi_{M}^{\lambda}(\zeta)=\sup \left\{\frac{1}{\lambda} \log |\psi(\zeta)|: \psi \in S \mathcal{H}_{M}^{\lambda}\right\} \\
\log \Phi_{M}=\lim \sup _{\lambda \rightarrow \infty} \log \Phi_{M}^{\lambda}
\end{array}\right.
$$

Remark:

One could define the analogous notion for any set $E \subset M_{\tau}$, with

$$
S \mathcal{H}_{E}^{\lambda}=\left\{p \in \mathcal{H}^{\lambda},\|p\|_{L^{2}(E)} \leq 1\right\} .
$$

But we only discuss the results for $E=M$ (see $\$ 10.3$ for comments on the general case).

One could also define the pluri-complex Green's function of $M_{\tau}$ as follows:

Definition 2. Let $(M, g)$ be a real analytic Riemannian manifold, let $M_{\tau}$ be an open Grauert tube, and let $E \subset M_{\tau}$. The Riemannian pluri-complex Green's function with respect to $\left(E, M_{\tau}, g\right)$ is defined by

$$
V_{g, E, \tau}(\zeta)=\sup \left\{u(z): u \in P S H\left(M_{\tau}\right),\left.u\right|_{E} \leq 0,\left.u\right|_{\partial M_{\tau}} \leq \tau\right\} .
$$

It is obvious that $V_{g, M, \tau}(\zeta) \geq \sqrt{\rho}(\zeta)$ and it is almost standard that $V_{g, M, \tau}(\zeta)=\sqrt{\rho}(\zeta)$. See Proposition 4.1 of [GZ] or Corollary 9 of [BT2]. The set $M=(\sqrt{\rho})^{-1}(0)$ is often called the center. As proved in [LS1, there are no smooth exhaustion functions solving the exact HCMA (Theorem 1.1). Hence $u$ must be singular on its minimum set. In [HW] it is proved that the minimum set of strictly PSH function is totally real. 
0.2. Statement of results. Our first results concern the logarithmic asymptotics of the complexified spectral projections.

TheOREM 1. (see also [Z4]) Let $I_{\lambda}=[0, \lambda]$. Then

(1) $\log \Phi_{M}^{\lambda}(\zeta)=\frac{1}{\lambda} \log \Pi_{I_{\lambda}}^{\mathbb{C}}(\zeta, \bar{\zeta})$.

(2) $\log \Phi_{M}=\lim _{\lambda \rightarrow \infty} \log \Phi_{M}^{\lambda}=\sqrt{\rho}$.

To prove the Theorem, it is convenient to study the tempered spectral projection measures (3), or in differentiated form,

$$
d_{\lambda} P_{[0, \lambda]}^{\tau}(\zeta, \bar{\zeta})=\sum_{j} \delta\left(\lambda-\lambda_{j}\right) e^{-2 \tau \lambda_{j}}\left|\varphi_{j}^{\mathbb{C}}(\zeta)\right|^{2}
$$

which is a temperate distribution on $\mathbb{R}$ for each $\zeta$ satisfying $\sqrt{\rho}(\zeta) \leq \tau$. When we set $\tau=\sqrt{\rho}(\zeta)$ we omit the $\tau$ and write

$$
d_{\lambda} P_{[0, \lambda]}(\zeta, \bar{\zeta})=\sum_{j} \delta\left(\lambda-\lambda_{j}\right) e^{-2 \sqrt{\rho}(\zeta) \lambda_{j}}\left|\varphi_{j}^{\mathbb{C}}(\zeta)\right|^{2} .
$$

The advantage of the tempered projections is that they have polynomial asymptotics and one can use standard Tauberian theorems to analyse their growth.

We prove the following one-term local Weyl law for complexified spectral projections:

THEOREM 2. On any compact real analytic Riemannian manifold $(M, g)$ of dimension $n$, we have, with remainders uniform in $\zeta$,

(1) For $\sqrt{\rho}(\zeta) \geq \frac{C}{\lambda}$

$$
P_{[0, \lambda]}(\zeta, \bar{\zeta})=(2 \pi)^{-n}\left(\frac{\lambda}{\sqrt{\rho}}\right)^{\frac{n-1}{2}}\left(\frac{\lambda}{(n-1) / 2+1}+O(1)\right) ;
$$

(2) For $\sqrt{\rho}(\zeta) \leq \frac{C}{\lambda}$

$$
P_{[0, \lambda]}(\zeta, \bar{\zeta})=(2 \pi)^{-n} \lambda^{n}\left(1+O\left(\lambda^{-1}\right)\right] .
$$

This implies new bounds on pointwise norms on complexified eigenfunctions, improving those of GLS]. inequality gives

Corollary 3. Suppose $(M, g)$ is real analytic of dimension $n$, and that $I_{\lambda}=[0, \lambda]$. Then,

(1) For $\tau \geq \frac{C}{\lambda}$ and $\sqrt{\rho}(\zeta)=\tau$, there exists $C>0$ so that

$$
C \lambda_{j}^{-\frac{n-1}{2}} e^{\tau \lambda} \leq \sup _{\zeta \in M_{\tau}}\left|\varphi_{\lambda}^{\mathbb{C}}(\zeta)\right| \leq C \lambda^{\frac{n-1}{4}+\frac{1}{2}} e^{\tau \lambda} .
$$

(2) For $\tau \leq \frac{C}{\lambda}$, and $\sqrt{\rho}(\zeta)=\tau$, there exists $C>0$ so that

$$
\left|\varphi_{\lambda}^{\mathbb{C}}(\zeta)\right| \leq \lambda^{\frac{n-1}{2}}
$$

The lower bound of Corollary 3 (1) combines Theorem 2 with Gärding's inequality. The upper bound sharpens the estimates claimed in [Bou, GLS],

$$
\sup _{\zeta \in M_{\tau}}\left|\varphi_{\lambda}^{\mathbb{C}}(\zeta)\right| \leq C_{\tau} \lambda^{n+1} e^{\tau \lambda}
$$

The improvement is due to using spectral asymptotics rather than a crude Sobolev inequality. 
The next Proposition ties together the work on triple inner products of eigenfunctions in $[\mathrm{Sar}, \mathrm{BR}$ ] and elsewhere with analytic continuations of eigenfunctions to Grauert tubes. The basic question is the decay rate of the inner products $\int_{M} \varphi_{\lambda_{j}} \varphi_{\lambda_{k}}^{2} d V_{g}$ where $d V_{g}$ is the volume form of $(M, g)$. More generally, one considers integrals where $\varphi_{\lambda_{k}}^{2}$ is replaced by a polynomial in eigenfunctions of fixed eigenvalues. In [Sar], it is proved that $\left|\left\langle P, \varphi_{\lambda_{k}}\right\rangle\right| \leq$ $A\left(\lambda_{k}+1\right)^{B} \exp \left(-\pi \sqrt{\lambda_{k}} / 2\right)$. The exponent is sharp, but the prefactor is improved in [BR]. The exponent constant $\frac{\pi}{2}$ is the radius of the maximal Grauert tube for hyperbolic space and its quotients (see $[\mathrm{Sz}, \mathrm{KM}]$ and $\$ 1.3$ for the latter fact). The next Proposition generalizes this bound to any real analytic metric. The radius $\tau_{a n}$ is the maximal analytic tube radius defined in Definition 1.1. Essentially, it is the largest tube to which all eigenfunctions analytically continue. Its relation to the geometric radius is discussed in $\$ 1.2$ and $\$ 11.1$.

Proposition 1. Let $(M, g)$ be any compact real analytic manifold and let $\tau_{a n}(g)$ be the maximal analytic Grauert tube radius. Then, for all $\tau<\tau_{\text {an }}$, there exists a constant $C_{\tau}$ such that

$$
\left|\int_{M} \varphi_{\lambda_{j}} \varphi_{\lambda_{k}}^{2} d V_{g}\right| \leq C_{\tau}\left(\lambda_{k}\right) e^{-\tau \lambda_{j}}
$$

If $\partial M_{\tau_{a n}(g)}$ is a smooth manifold and $\varphi_{\lambda_{k}}^{\mathbb{C}}$ is a distribution of order $r$ on $\partial M_{\tau_{a n}(g)}$, then there exists a constant $C$ so that

$$
\left|\int_{M} \varphi_{\lambda_{j}} \varphi_{\lambda_{k}}^{2} d V_{g}\right| \leq C\left(\lambda_{k}\right) \lambda_{j}^{r} e^{-\tau_{a n}(g) \lambda_{j}}
$$

As will be seen in the proof, $C\left(\lambda_{k}\right)$ is a Sobolev $W_{s}$ norm of $e^{\tau \sqrt{\Delta}} \varphi_{\lambda_{k}}$. The statement lacks the precision of the hyperbolic case, since we do not determine whether $\partial M_{\tau}$ is even a smooth manifold. In \$11.1, we sketch a proof that $\tau_{a n}$ is the usual geometric radius of the Grauert tube, and then the estimate of Proposition 1 has almost the same exponential asymptotics as in the hyperbolic case.

Finally, we give an application to complex zeros of the joint eigenfunctions of the algebra $\mathcal{D}$ of invariant differential operators on the locally symmetric space $S O(n, \mathbb{R}) \backslash S L_{n}(\mathbb{R}) / \Gamma$, where $\Gamma$ is a co-compact discrete subgroup of $S L_{n}(\mathbb{R})$ In [AS], Anantharaman-Silberman proved a number of results on the entropies of the quantum limit measures of the joint eigenfunctions as the joint eigenvalue tends to infinity. Roughly speaking, the results say that the quantum limit measures must have a non-trivial Haar component. This result is sufficient to determine the limit distribution of complex zeros of the complexifications of the same eigenfunctions. We denote by $\left[Z_{\varphi_{\lambda}^{\mathbb{C}}}\right]$ the current of integration over the complex zero set of $\varphi_{\lambda}^{\mathbb{C}}$.

TheOREM 4. Let $(M, g)$ be a compact locally symmetric manifold, and let $\left\{\varphi_{\lambda}\right\}$ be any orthonormal basis of the joint $\mathcal{D}$-eigenfunctions. Then for $\tau<\tau_{a n}$.

$$
\frac{1}{\lambda}\left[Z_{\varphi_{\lambda}^{\mathbb{C}}}\right] \rightarrow \frac{i}{\pi} \partial \bar{\partial} \sqrt{\rho}, \text { weakly in } \mathcal{D}^{\prime(1,1)}\left(M_{\tau}\right),
$$

for the entire sequence of eigenfunctions $\left\{\psi_{j}\right\}$.

This proof requires just a small observation improving on the weak convergence result of [Z3], placed on top of the very strong Haar component theorem of Anantharaman-Silberman. 
0.3. Results of [Z1]. The asymptotics of the complexified spectral projection kernels (2) are complex analogues of those of the diagonal spectral projections in the real domain and reflect the structure of complex geodesics from $\zeta$ to $\bar{\zeta}$. As in the real domain, one can obtain more refined asymptotics of $P_{[\lambda, \lambda+1]}(\zeta, \bar{\zeta})$ by using the structure of geodesic segments from $\zeta$ to $\bar{\zeta}$. This is the subject of the sequel [Z1]. For the sake of completeness, we state the results here: There exists an explicit complex oscillatory factor $Q_{\zeta}(\lambda)$ depending on the geodesic arc from $\zeta$ to $\bar{\zeta}$ such that

(1) For $\sqrt{\rho}(\zeta) \geq \frac{C}{\lambda}$,

$$
P_{[0, \lambda]}^{\tau}(\zeta, \bar{\zeta})=(2 \pi)^{-n} \lambda\left(\frac{\lambda}{\sqrt{\rho}}\right)^{\frac{n-1}{2}}\left(1+Q_{\zeta}(\lambda) \lambda^{-1}+o\left(\lambda^{-1}\right)\right) ;
$$

(2) For $\sqrt{\rho}(\zeta) \leq \frac{C}{\lambda}$

$$
P_{[0, \lambda]}^{\tau}(\zeta, \bar{\zeta})=(2 \pi)^{-n} \lambda^{n}+Q_{\zeta}(\lambda) \lambda^{n-1}+o\left(\lambda^{n-1}\right),
$$

The functions $Q_{\zeta}(\lambda)$ depend on whether $(M, g)$ is a manifold without conjugate points, or with conjugate points. We refer to [Z1] for the formulae. A special case is that of Zoll manifolds where there exists a complete asymptotic expansion similar to that for line bundles. The two term asymptotics lead to improvement by one order of magnitude on the bounds in Corollary [3, and are sharp in that they are achieved by complexified zonal spherical harmonics on a standard sphere.

\section{Grauert tubes AND COMPlex GeOdesic FlOW}

By a theorem of Bruhat-Whitney, a real analytic Riemannian manifold $M$ admits a complexification $M_{\mathbb{C}}$, i.e. a complex manifold into which $M$ embeds as a totally real submanifold. Corresponding to a real analytic metric $g$ is a unique plurisubharmonic exhaustion function $\sqrt{\rho}$ on $M_{\mathbb{C}}$ satisfying two conditions (i) It satisfies the Monge-Ampère equation $(i \partial \bar{\partial} \sqrt{\rho})^{n}=\delta_{M, g}$ where $\delta_{M, g}$ is the delta function on $M$ with density $d V_{g}$ equal to the volume density of $g$; (ii) the Kähler metric $\omega_{g}=i \partial \bar{\partial} \rho$ on $M_{\mathbb{C}}$ agrees with $g$ along $M$. In fact,

$$
\sqrt{\rho}(\zeta)=\frac{1}{2 i} \sqrt{r_{\mathbb{C}}^{2}(\zeta, \bar{\zeta})}
$$

where $r^{2}(x, y)$ is the square of the distance function and $r_{\mathbb{C}}^{2}$ is its holomorphic extension to a small neighborhood of the anti-diagonal $(\zeta, \bar{\zeta})$ in $M_{\mathbb{C}} \times M_{\mathbb{C}}$. In the case of flat $\mathbb{R}^{n}$, $\sqrt{\rho}(x+i \xi)=2|\xi|$ and in general $\sqrt{\rho}(\zeta)$ measures how far $\zeta$ reaches into the complexification of $M$. The open Grauert tube of radius $\tau$ is defined by $M_{\tau}=\left\{\zeta \in M_{\mathbb{C}}, \sqrt{\rho}(\zeta)<\tau\right\}$. We use the imprecise notation $M_{\mathbb{C}}$ to denote the open complexificaiton when it is not important to specify the radius.

1.1. Analytic continuation of the exponential map. The geodesic flow is a Hamiltonian flow on $T^{*} M$. In fact, there are two standard choices of the Hamiltonian. In PDE it is most common to define the (real) homogeneous geodesic flow $g^{t}$ of $(M, g)$ as the Hamiltonian flow on $T^{*} M$ generated by the Hamiltonian $|\xi|_{g}$ with respect to the standard Hamiltonian form $\omega$. This Hamiltonian is real analytic on $T^{*} M \backslash 0$. In Riemannian geometry it is standard to let the time of travel equal $|\xi|_{g}$; this corresponds to the Hamiltonian flow of $|\xi|_{g}^{2}$, which is real analytic on all of $T^{*} M$. We denote its Hamiltonian flow by $G^{t}$. In general, we denote 
by $\Xi_{H}$ the Hamiltonian vector field of a Hamiltonian $H$ and its flow by $\exp t \Xi_{H}$. Both of the Hamiltonian flows

- $g^{t}=\exp t \Xi_{|\xi|_{g}}$;

- $G^{t}=\exp t \Xi_{|\xi|_{g}^{2}}$

are important in analytic continuation of the wave kernel. The exponential map is the map $\exp _{x}: T^{*} M \rightarrow M$ defined by $\exp _{x} \xi=\pi G^{t}(x, \xi)$ where $\pi$ is the standard projection.

We denote by $\operatorname{inj}(x)$ the injectivity radius of $(M, g)$ at $x$, i.e. the radius $r$ of the largest ball on which $\exp _{x}: B_{r} M \rightarrow M$ is a diffeomorphism to its image. Since $(M, g)$ is real analytic, $\exp _{x} t \xi$ admits an analytic continuation in $t$ and the imaginary time exponential map

$$
E: B_{\varepsilon}^{*} M \rightarrow M_{\mathbb{C}}, \quad E(x, \xi)=\exp _{x} i \xi
$$

is, for small enough $\varepsilon$, a diffeomorphism from the ball bundle $B_{\varepsilon}^{*} M$ of radius $\varepsilon$ in $T^{*} M$ to the Grauert tube $M_{\varepsilon}$ in $M_{\mathbb{C}}$. We have $E^{*} \omega=\omega_{T^{*} M}$ where $\omega=i \partial \bar{\partial} \rho$ and where $\omega_{T^{*} M}$ is the canonical symplectic form; and also $E^{*} \sqrt{\rho}=|\xi|$ [GS1, LS1]. It follows that $E^{*}$ conjugates the geodesic flow on $B^{*} M$ to the Hamiltonian flow $\exp t \Xi_{\sqrt{\rho}}$ of $\sqrt{\rho}$ with respect to $\omega$, i.e.

$$
E\left(g^{t}(x, \xi)\right)=\exp t \Xi_{\sqrt{\rho}}\left(\exp _{x} i \xi\right) .
$$

1.2. Maximal Grauert tubes. A natural definition of maximal Grauert tube is the maximum value of $\varepsilon$ so that (9) is a diffeomorphism. We refer to this radius as the maximal geometric tube radius. But for purposes of this paper, another definition of maximality is relevant: the maximal tube on which all eigenfunctions extend holomorphically. A closely related definition is the maximal tube to which the Poisson kernel (32) extends holomorphically. We refer to the radius as the maximal analytic tube radius.

A natural question is to relate these notions of maximal Grauert tube has not been explored. We therefore define the radii more precisely:

DEFINITION 1.1. (1) The maximal geometric tube radius $\tau_{g}$ is the largest radius $\varepsilon$ for which $E$ (9) is a diffeomorphism.

(2) The maximal analytic tube radius $\tau_{a n} M_{\tau_{a n}} \subset M_{\mathbb{C}}$ is the maximal tube to which all eigenfunctions extend holomorphically and to which the anti-diagonal $U(2 i \tau, \zeta, \bar{\zeta})$ of the Poisson kernel admits an analytic continuation.

We make:

CONJECTURE 1. $\tau_{g}=\tau_{a n}$.

In $\$ 11.1$ we sketch the proof, which is based on holomorphic extensions of solutions of analytic PDE across non-characteristic hypersurfaces. It would require too much background to incude a more detailed proof here, but we hope the sketch of proof indicates the main ideas. We found a similar argument in [KS] in the case of locally symmetric spaces but employing additional arguments. We intend to give more details in [Z5].

\subsection{Model examples. .}

We consider some standard examples to clarify these analytic continuations.

\section{(i) Complex tori:}

The complexification of the torus $M=\mathbb{R}^{m} / \mathbb{Z}^{m}$ is $M_{\mathbb{C}}=\mathbb{C}^{m} / \mathbb{Z}^{m}$. The adapted complex structure to the flat metric on $M$ is the standard (unique) complex structure on $\mathbb{C}^{m}$. The 
complexified exponential map is $\exp _{x}^{\mathbb{C}}(i \xi)=z:=x+i \xi$, while the distance function $r(x, y)=$ $|x-y|$ extends to $r_{\mathbb{C}}(z, w)=\sqrt{(z-w)^{2}}$. Then $\sqrt{\rho}(z, \bar{z})=\sqrt{(z-\bar{z})^{2}}= \pm 2 i|\operatorname{Im} z|= \pm 2 i|\xi|$.

The complexified cotangent bundle is $T^{*} M_{\mathbb{C}}=\mathbb{C}^{m} / \mathbb{Z}^{m} \times \mathbb{C}^{m}$, and the holomorphic geodesic flow is the entire holomorphic map

$$
G^{t}\left(\zeta, p_{\zeta}\right)=\left(\zeta+t p_{\zeta}, p_{\zeta}\right)
$$

(ii) $\mathbb{S}^{n}$ GS1 The unit sphere $x_{1}^{2}+\cdots+x_{n+1}^{2}=1$ in $\mathbb{R}^{n+1}$ is complexified as the complex quadric

$$
\mathbb{S}_{\mathbb{C}}^{n}=\left\{\left(z_{1}, \ldots, z_{n}\right) \in \mathbb{C}^{n+1}: z_{1}^{2}+\cdots+z_{n+1}^{2}=1\right\} .
$$

If we write $z_{j}=x_{j}+i \xi_{j}$, the equations become $|x|^{2}-|\xi|^{2}=1,\langle x, \xi\rangle=0$. The geodesic flow $\left.G^{t}(x, \xi)=(\cos t|\xi|) x+(\sin t|\xi|) \frac{\xi}{|\xi|},-|\xi|(\sin t|\xi|) x+(\cos t|\xi|) \xi\right)$ on $T^{*} \mathbb{S}^{n}$ complexifies to

$$
\begin{aligned}
G^{t}(Z, W) & =(\cos t \sqrt{W \cdot W}) Z+(\sin t \sqrt{W \cdot W})) \frac{W}{\sqrt{W \cdot W})}, \\
& -\sqrt{W \cdot W})(\sin t \sqrt{W \cdot W})) Z+(\cos t \sqrt{W \cdot W})) W), \quad\left((Z, W) \in T^{*} \mathbb{S}_{\mathbb{C}}^{m}\right) .
\end{aligned}
$$

Here, the real cotangent bundle is the subset of $T^{*} \mathbb{R}^{n+1}$ of $(x, \xi)$ such that $x \in \mathbb{S}^{n}, x \cdot \xi=0$ and the complexified cotangent bundle $T^{*} \mathbb{S}_{\mathbb{C}}^{n} \subset T^{*} \mathbb{C}^{n+1}$ is the set of vectors $(Z, W): Z$. $W=0$. We note that although $\sqrt{W \cdot W}$ is singular at $W=0$, both $\cos \sqrt{W \cdot W}$ ) and $\sqrt{W \cdot W}) \sin t \sqrt{W \cdot W}$ ) are holomorphic. The Grauert tube function equals

$$
\sqrt{\rho}(z)=i \cosh ^{-1}|z|^{2}, \quad\left(z \in \mathbb{S}_{\mathbb{C}}^{n}\right) .
$$

It is globally well defined on $\mathbb{S}_{\mathbb{C}}^{n}$. The characteristic conoid is defined by $\cosh \frac{1}{i} \sqrt{\rho}=\cosh \tau$.

(iii) (See e.g. $[\mathrm{KM}]$ ). $\mathbb{H}^{n}$ The hyperboloid model of hyperbolic space is the hypersurface in $\mathbb{R}^{n+1}$ defined by

$$
\mathbb{H}^{n}=\left\{x_{1}^{2}+\cdots x_{n}^{2}-x_{n+1}^{2}=-1, \quad x_{n}>0\right\} .
$$

Then,

$$
H_{\mathbb{C}}^{n}=\left\{\left(z_{1}, \ldots, z_{n+1}\right) \in \mathbb{C}^{n+1}: z_{1}^{2}+\cdots z_{n}^{2}-z_{n+1}^{2}=-1\right\} .
$$

In real coordinates $z_{j}=x_{j}+i \xi_{j}$, this is:

$$
\langle x, x\rangle_{L}-\langle\xi, \xi\rangle_{L}=-1, \quad\langle x, \xi\rangle_{L}=0
$$

where $\langle,\rangle_{L}$ is the Lorentz inner product of signature $(n, 1)$. Hence the complexified hyperbolic space is the hypersurface in $\mathbb{C}^{n+1}$ given by the same equations.

We obtain $\mathbb{H}_{\mathbb{C}}^{n}$ from $\mathbb{S}_{\mathbb{C}}^{n}$ by the map $\left(z^{\prime}, z_{n+1}\right) \rightarrow\left(i z^{\prime}, z_{n+1}\right)$. The complexified geodesic flow is given by

$$
\begin{aligned}
G^{t}(Z, W) & =\left(\cosh t \sqrt{\langle W, W\rangle_{L}} Z+\left(\sinh t \sqrt{\langle W, W\rangle_{L}}\right)\right) \frac{W}{\sqrt{\langle W, W\rangle_{L}}}, \\
& \left.\left.\left.\left.-\sqrt{\langle W, W\rangle_{L}}\right)\left(\sinh t \sqrt{\langle W, W\rangle_{L}}\right)\right) Z+\left(\cosh t \sqrt{\langle W, W\rangle_{L}}\right)\right) W\right), \quad\left((Z, W) \in T^{*} \mathbb{H}^{m}\right) .
\end{aligned}
$$

The Grauert tube function is:

$$
\sqrt{\rho}(z)=\cos ^{-1}\left(\|x\|_{L}^{2}+\|\xi\|_{L}^{2}-\pi\right) / \sqrt{2} .
$$

The radius of maximal Grauert tube is $\varepsilon=1$ or $r=\pi / \sqrt{2}$. 


\section{2. $\Delta_{g}, \square_{g}$ AND CHARACTERISTICS}

The Laplacian of $(M, g)$ is given in local coordinates by

$$
\Delta_{g}=-\frac{1}{\Theta} \sum_{i, j} \frac{\partial}{\partial x_{i}}\left(\Theta g^{i j}\right) \frac{\partial}{\partial x_{j}}
$$

is the Laplacian of $(M, g)$. Here, $g_{i j}=g\left(\frac{\partial}{\partial x_{i}}, \frac{\partial}{\partial x_{j}}\right),\left[g^{i j}\right]$ is the inverse matrix to $\left[g_{i j}\right]$ and $\Theta=\sqrt{\operatorname{det}\left[g_{i j}\right]}$. Since $g$ is fixed we henceforth write the Laplacian as $\Delta$. Note that we have put a minus sign in front of the sum of squares to make $\Delta$ a non-negative operator. This is for later notational convenience. On a compact manifold, $\Delta$ is negative operator with discrete spectrum

$$
\Delta \varphi_{j}=\lambda_{j}^{2} \varphi_{j}, \quad\left\langle\varphi_{j}, \varphi_{k}\right\rangle=\delta_{j k}
$$

of eigenvalues and eigenfunctions. Note that the eigenvalues are denoted $\lambda_{j}^{2}$; we refer to $\lambda_{j}$ as the 'frequency'.

In the real domain, $\Delta$ is an elliptic operator with principal symbol $\sigma_{\Delta}(x, \xi)=: \sum_{i, j=1}^{n} g^{i j}(x) \xi_{i} \xi_{j}$. Hence its characteristic set (the zero set of its symbol) consists only of the zero section $\xi=0$ in $T^{*} M$. But when we continue it to the complex domain it develops a complex characteristic set

$$
\operatorname{Ch}\left(\Delta_{\mathbb{C}}\right)=\left\{(\zeta, \xi) \in T^{*} M_{\mathbb{C}}: \sum_{i, j=1}^{n} g^{i j}(\zeta) \xi_{i} \xi_{j}=0\right\} .
$$

The wave operator on the product spacetime $\left(\mathbb{R} \times M, d t^{2}-g_{x}\right)$ is given by

$$
\square_{g}=\frac{\partial^{2}}{\partial t^{2}}+\Delta_{g}
$$

The unusual sign in front of $\Delta_{g}$ is due to the sign normalization above making the Laplacian non-negative. Again we omit the subscript when the metric is fixed. The characteristic variety of $\square$ is the zero set of its symbol

$$
\sigma_{\square}(t, \tau, x, \xi)=\tau^{2}-|\xi|_{x}^{2},
$$

that is,

$$
\mathrm{Ch}(\square)=\left\{(t, \tau, x, \xi) \in T^{*}(\mathbb{R} \times M): \tau^{2}-|\xi|_{x}^{2}=0\right\} .
$$

The null-bicharacteristic flow of $\square$ is the Hamiltonian flow of $\tau^{2}-|\xi|_{x}^{2}$ on $\mathrm{Ch}(\square)$. Its graph is thus

$$
\Lambda=\left\{(t, \tau, x, \xi, y, \eta): \tau^{2}-|\xi|_{x}^{2}=0, G^{t}(x, \xi)=(y, \eta)\right\} \subset T^{*}(\mathbb{R} \times M \times M) .
$$

2.1. Characteristic variety and characteristic conoid. Following $[\mathrm{H}]$, we put

$$
\Gamma(t, x, y)=t^{2}-r^{2}(x, y) .
$$

Here, $r(x, y)$ is the distance between $x, y$. It is singular at $r=0$ and also when $\mathrm{y}$ is in the "cut locus" of $x$. In this article we only consider $(x, y)$ so that $r(x, y)<\operatorname{inj}(x)$, where inj(x) is the injectivity radius at $x$, i.e. is the largest $\varepsilon$ so that

$$
\exp _{x}: B_{x, \varepsilon}^{*} M \rightarrow M
$$


is a diffeomorphism to its image. The injectivity radius $\operatorname{inj}(M, g)$ is the maximum of $\operatorname{inj}(x)$ for $x \in M$. Thus, we work in a sufficiently small neighborhood of the diagonal so that cut points do not occur.

The squared distance $r^{2}(x, y)$ is smooth in a neighborhoof of the diagonal. On a simply connected manifold $(\tilde{M}, g)$ without conjugate points, it is globally smooth on $\tilde{M} \times \tilde{M}$. We recall that 'without conjugate points' means that $\exp _{x}: T_{x} M \rightarrow M$ is non-singular for all $x$.

The characteristic conoid is the set

$$
\mathcal{C}=\left\{(t, x, y): r(x, y)<\operatorname{inj}(x), r^{2}(x, y)=t^{2}\right\} \subset \mathbb{R} \times M \times M .
$$

It separates $\mathbb{R} \times M \times M$ into the forward/backward semi-cones

$$
\mathcal{C}_{ \pm}=\left\{(t, x, y): t^{2}-r^{2}(x, y)>0, \pm t>0\right\}
$$

The complexificationof $\mathcal{C}=\mathcal{C}_{\mathbb{R}}$ is the complex characteristic conoid

$$
\mathcal{C}_{\mathbb{C}}=\left\{(t, x, y): r_{\mathbb{C}}^{2}(x, y)=t^{2}\right\} \subset \mathbb{C} \times M_{\mathbb{C}} \times M_{\mathbb{C}}
$$

We note that $\mathcal{C}_{\mathbb{R}} \subset \mathcal{C}_{\mathbb{C}}$ is a totally real submanifold. Another totally real submanifold of central importance in this article is the 'diagonal' (or anti-diagonal) conoid,

$$
\mathcal{C}_{\Delta}=\left\{(2 i \tau, \zeta, \bar{\zeta}): \tau \in \mathbb{R}_{+}, \zeta, \bar{\zeta} \in \partial M_{\tau}\right\}
$$

By definition, $r_{\mathbb{C}}^{2}(\zeta, \bar{\zeta})=-4 \tau^{2}$ if $\zeta \in \partial M_{\tau}$.

\section{Propagators and Fundamental solutions}

The main 'wave kernels' in this article are the half-wave kernel $e^{i t \sqrt{\Delta}}$ and the Poisson kernel $e^{-\tau \sqrt{\Delta}}$ for $\tau>0$. To put these kernels into context, we now give a brief review of propagators and fundamental solutions for the wave equation. We use the term 'propagator' for a solution operator to a Cauchy problem. It will be a homogeneous solution of $\square E=0$ with special initial conditions. We use the term 'fundamental solution' for a solution of the inhomogeneous equation $\square E=\delta_{0}$. We freely use standard notation for homogeneous distributions on $\mathbb{R}$ and refer to $[\mathrm{Ho}]$ for notation and background.

3.1. Cauchy problem for the wave equation. The Cauchy problem for the wave equation on $\mathbb{R} \times M$ is the initial value problem (with Cauchy data $f, g$ )

$$
\left\{\begin{array}{l}
\square u(t, x)=0, \\
u(0, x)=f, \quad \frac{\partial}{\partial t} u(0, x)=g(x),
\end{array}\right.
$$

The solution operator of the Cauchy problem (the "propagator") is the wave group,

$$
\mathcal{U}(t)=\left(\begin{array}{cc}
\cos t \sqrt{\Delta} & \frac{\sin t \sqrt{\Delta}}{\sqrt{\Delta}} \\
\sqrt{\Delta} \sin t \sqrt{\Delta} & \cos t \sqrt{\Delta}
\end{array}\right) .
$$

The solution of the Cauchy problem with data $(f, g)$ is $\mathcal{U}(t)\left(\begin{array}{l}f \\ g\end{array}\right)$.

Two of the components of $\mathcal{U}(t)$ are particularly important: 
- The even part $\cos t \sqrt{\Delta}$ is the solution operator of the initial value problem,

$$
\left\{\begin{array}{l}
\square u=0 \\
\left.u\right|_{t=0}=\left.f \quad \frac{\partial}{\partial t} u\right|_{t=0}=0
\end{array}\right.
$$

- The odd part $\frac{\sin t \sqrt{\Delta}}{\sqrt{\Delta}}$ is the solution operator of the initial value problem,

$$
\left\{\begin{array}{l}
\square u=0 \\
\left.u\right|_{t=0}=\left.0 \quad \frac{\partial}{\partial t} u\right|_{t=0}=g
\end{array}\right.
$$

The kernels of $\cos t \sqrt{\Delta}, \frac{\sin t \sqrt{\Delta}}{\sqrt{\Delta}}$ exhibit finite propagation speed of solutions of the wave equation, i.e. are supported inside the characteristic conoid $\mathcal{C}$ where $r \leq|t|$.

3.2. Cauchy problem for the half-wave equation. The forward half-wave group is the solution operator of the Cauchy problem

$$
\left(\frac{1}{i} \frac{\partial}{\partial t}-\sqrt{\Delta}\right) u=0, \quad u(0, x)=u_{0} .
$$

The solution is given by

$$
u(t, x)=U(t) u_{0}(x), \text { with } U(t)=e^{i t \sqrt{\Delta}} .
$$

The Schwartz kernel $U(t, x, y)$ of the wave group $U(t)=e^{i t \sqrt{\Delta}}$ solves the pseudo-differential Cauchy problem(the half-wave equation),

$$
\left(\frac{1}{i} \frac{\partial}{\partial t}-{\sqrt{\Delta_{x}}}\right) U(t, x, y)=0, \quad U(0, x, y)=\delta_{y}(x) .
$$

Equivalently, it solves the wave equation with pseudo-differential initial condition,

$$
\left\{\begin{array}{l}
\square U=0, \\
U(0, x, y)=\delta_{y}(x),\left.\quad \frac{\partial}{\partial t} U(t, x, y)\right|_{t=0}=i{\sqrt{\Delta_{x}}}_{x} \delta_{x}(y)
\end{array}\right.
$$

The solution is given by

$$
U(t, x, y)=\cos t \sqrt{\Delta}(x, y)+i \sqrt{\Delta}_{x} \frac{\sin t \sqrt{\Delta}}{\sqrt{\Delta}}(x, y) .
$$

Unlike the even/odd kernels, $e^{i t \sqrt{\Delta}}$ has infinite propagation speed, i.e. is non-zero outside the characteristic conoid $\mathcal{C}$; this is due to the second of its initial condition.

The half wave group has the eigenfunction expansion,

$$
U(t, x, y)=\sum_{j} e^{i t \lambda_{j}} \varphi_{\lambda_{j}}(x) \varphi_{\lambda_{j}}(y)
$$

on $\mathbb{R} \times M \times M$, which converges in the sense of distributions. 
3.3. Fundamental solutions. A fundamental solution of the wave equation is a solution of

$$
\square E(t, x, y)=\delta_{0}(t) \delta_{x}(y) .
$$

The right side is the Schwartz kernel of the identity operator on $\mathbb{R} \times M$.

There exists a unique fundamental solution which is supported in the forward conoid

$$
C_{+}=\left\{(t, x, y): t>0, t^{2}-r^{2}(x, y)>0\right\} .
$$

called the advanced (or forward) propagator. It is given by

$$
E_{+}(t)=H(t) \frac{\sin t \sqrt{\Delta}}{\sqrt{\Delta}},
$$

where $H(t)=\mathbf{1}_{t \geq 0}$ is the Heaviside step function. It is well-defined for any curved globally hyperbolic spacetime, while Cauchy problems and propagators require a choice of "Cauchy hypersurface" like $\{t=0\}$.

In $[R]$, the forward fundamental solution is constructed in terms of the holomorphic family of Riesz kernels $\frac{\left(t^{2}-r^{2}\right)_{+}^{\alpha}}{\Gamma(\alpha+1)}$, which are supported in the forward characteristic conoid $\mathcal{C}_{+}$. A more contemporary treatment using the language of homogeneous distributions on $\mathbb{R}$ is given in $[\mathrm{Be}$. In [J] it is pointed out that the Riesz kernels are Schwartz kernels of complex powers $\square^{\alpha}$ of the wave operator on $\mathbb{R} \times M$. Unlike complex powers of $\Delta, \square^{\alpha}$ is only uniquely defined if the Scwhartz kernels are assumed to be supported in $\mathcal{C}_{+}$.

3.4. Hadamard-Feynman fundamental solution. Hadamard and Feynman constructed another fundamental solution which is a (branched) meromorphic function of $(t, x, y)$ near the characteristic conoid with the singularity $\left(t^{2}-r^{2}\right)^{-\frac{m-2}{2}}$ analogous to the Newtonian potential $r^{2-n}$ in the elliptic case (here $m=n+1=\operatorname{dim} \mathbb{R} \times M^{n}$.) and is not supported in $\mathcal{C}_{+}$. It corresponds to the inverse $(\square+i 0)^{-1}$ rather than to the Riesz kernel $\square^{-1}$. It is the

For background in the case of $\mathbb{R}^{n} \times \mathbb{R}$ we refer to [IZ] and for the general case we refer to $[\mathrm{DH}]$. Hadamard $[\mathrm{H}]$ defined this fundamental solution to be the branched meromorphic fundamental solution of $\square$, and referred to it as the 'elementary solution. We review his parametrix extensively in $\$ 4$.

Definition 3.1. The Hadamard-Feynman fundamental solution is the operator

$$
(\square+i 0)^{-1}=\int_{\mathbb{R}} e^{i t \tau}\left(\Delta-\tau^{2}+i 0\right)^{-1} d t
$$

on $\mathbb{R} \times M$.

Proposition 3.2. As a family $U_{F}(t)$ of operators on $L^{2}(M)$ it is given by

$$
U_{F}(t)=\frac{e^{i|t| \sqrt{\Delta}}}{\sqrt{\Delta}} .
$$

Proof. The proof is essentially the same as in the case of $M=\mathbb{R}^{n}$ (see for instance [IZ]). Using the eigenfunction expansion

$$
\left(\Delta-\tau^{2}+i 0\right)^{-1}=\sum_{j}\left(\lambda^{2}-\tau^{2}+i 0\right)^{-1} \varphi_{j}(x) \varphi_{j}(y)
$$


it suffices to show that

$$
\int_{\mathbb{R}} e^{i t \tau}\left(\lambda_{j}^{2}-\tau^{2}+i 0\right)^{-1} d \tau=\frac{e^{i|t| \lambda_{j}}}{\lambda_{j}} .
$$

The evaluation follows by a residue calculation.

Remark: One can verify that $U_{F}(t)$ is a fundamental solution directly by applying $\square$. by

The analogous expressions for the advanced (resp. retarded) Green's function are given

$$
G_{r e t}(t, x, y)=-\sum_{j}\left(\int_{\mathbb{R}} e^{-i t \tau}\left((\tau+i \varepsilon)^{2}-\lambda_{j}^{2}\right)^{-1} d \tau\right) \varphi_{j}(x) \varphi_{j}(y)
$$

Since

$$
\left((\tau+i \varepsilon)^{2}-\lambda_{j}^{2}\right)^{-1}=\frac{1}{2 \lambda_{j}}\left(\frac{1}{\tau-\lambda_{j}+i \varepsilon}-\frac{1}{\tau+\lambda_{j}+i \varepsilon}\right)
$$

we have

$$
G_{r e t}(t, x, y)=C_{n} H(t) \sum_{j} \frac{\sin t \lambda_{j}}{\lambda_{j}} \varphi_{j}(x) \varphi_{j}(y) .
$$

3.5. Fundamental solutions and half-wave propagator on $\mathbb{R}^{n}$. We illustrate the definitions in the case of $\mathbb{R}^{n}$ following [Ho]. We use the notation $\chi_{+}^{\alpha}(x)=\frac{x_{+}^{\alpha}}{\Gamma(\alpha+1)}$. The advanced/retarded fundamental solutions of $\square$ on $\mathbb{R}^{n+1}=\mathbb{R}_{t} \times \mathbb{R}_{x}^{n}$ is given by

$$
E_{ \pm}(t, x, y)=\chi_{ \pm}^{\frac{2-(n+1)}{2}}(\Gamma)
$$

where we use the Hadamard notation (13) (which unfortunately clashes with the Gamma function).

The Hadamard-Feynamn fundamental solution on $\mathbb{R}^{n+1}$ is a ramified (branched) holomorphic fundamental solution

$$
U_{F}(t, x, y)=(\Gamma+i 0)^{\frac{2-(n+1)}{2}} .
$$

There is an associated fundamental solution corresponding to $(\Gamma-i 0)^{\frac{2-(n+1)}{2}}$.

The half-wave propagator is constructed on $\mathbb{R}^{n}$ by the Fourier inversion formula,

$$
U(t, x, y)=\int_{\mathbb{R}^{n}} e^{i\langle x-y, \xi\rangle} e^{i t|\xi|} d \xi
$$

The Poisson kernel (extending functions on $\mathbb{R}^{n}$ to harmonic functions on $\mathbb{R}_{+} \times \mathbb{R}^{n}$ ) is the half-wave propagagor at positive imaginary times $t=i \tau(\tau>0)$,

$$
\begin{aligned}
U(i \tau, x, y) & =\int_{\mathbb{R}^{n}} e^{i\langle x-y, \xi\rangle} e^{-\tau|\xi|} d \xi \\
& \left.=\tau^{-n}\left(1+\left(\frac{x-y}{\tau}\right)^{2}\right)^{-\frac{n+1}{2}}=\tau\left(\tau^{2}+(x-y)^{2}\right)\right)^{-\frac{n+1}{2}} .
\end{aligned}
$$

In the case of $\mathbb{R}^{n}$, the Poisson kernel analytically continues to $t+i \tau, \zeta=x+i p \in \mathbb{C}_{+} \times \mathbb{C}^{n}$ as the integral

$$
U(t+i \tau, x+i p, y)=\int_{\mathbb{R}^{n}} e^{i(t+i \tau)|\xi|} e^{i\langle\xi, x+i p-y\rangle} d \xi
$$


which converges absolutely for $|p|<\tau$. If we substitute $\tau \rightarrow \tau-i t$ and let $\tau \rightarrow 0$ we get the formula

$$
\left.U(t, x, y)=C_{n} \lim _{\tau \rightarrow 0} i t((t+i \tau))^{2}-r(x, y)^{2}\right)^{-\frac{n+1}{2}}
$$

for a constant $C_{n}$ depending only on the dimension.

\section{Remark:}

We observe that the half-wave kernel differs from the Hadamard-Feynman fundamental solution not only in the power but also because the former uses powers of the quadratic form $\left((t+i 0)^{2}-r^{2}\right)$ while the latter uses $\left(t^{2}-r^{2}+i 0\right)$. That is, with $r^{2}=|x-y|^{2}$,

- $C_{n}\left(t^{2}-r^{2}+i 0\right)^{-\frac{n-1}{2}}$ is the Feynman fundamental solution.

- $C_{n}^{\prime} t\left((t+i 0)^{2}-r^{2}\right)^{-\frac{n+1}{2}}$ is the solution operator kernel of the half-wave equation.

This difference in the kernels holds for general $(M, g)$. In the half-wave kernel, $(t+i \varepsilon)^{2}=$ $t^{2}-\varepsilon^{2}+2 i t \varepsilon$ and the imaginary part only has a fixed sign if we assume that $t>0$. This in part explains the $|t|$-dependence in the formula of Proposition 3.2 .

We observe that neither kernel has 'finite propagation speed', i.e. neither is supported in the characteristic conoid.

3.6. Subordination of the Poisson kernel to the heat kernel. There is another standard approach to the Poisson kernel based on the 'subordination identity'

$$
e^{-\gamma}=\frac{1}{\sqrt{\pi}} \int_{0}^{\infty} \frac{e^{-u}}{\sqrt{u}} e^{-\frac{\gamma^{2}}{4 u}} d u
$$

More generally, for any positive operator $A$,

$$
e^{-t A}=\frac{t}{2 \sqrt{\pi}} \int_{0}^{\infty} e^{-\frac{t^{2}}{4 u}} e^{-u A^{2}} u^{-3 / 2} d u
$$

This recuces the construction of the Poisson kernel to the heat kernel, which is useful since there exists a well-known parametrix for the heat kernel (Levi, Minakshisundaram-Pleijel). We follow the exposition in [St] $\S I I I .2$.

The subordination identity follows from two further identities:

$$
\text { (i) } \int_{\mathbb{R}^{n}} e^{-\pi \delta|t|^{2}} e^{-2 \pi i\langle t, x\rangle} d t=\delta^{-n / 2} e^{-\pi \frac{|x|^{2}}{\delta}},
$$

and

$$
\text { (ii) } e^{-\gamma}=\frac{1}{\sqrt{\pi}} \int_{0}^{\infty} \frac{e^{-u}}{\sqrt{u}} e^{-\frac{\gamma^{2}}{4 u}} d u
$$

Proof of (ii): We have

$$
e^{-\gamma}=\frac{1}{\sqrt{\pi}} \int_{-\infty}^{\infty} \frac{e^{i \gamma \cdot x}}{1+x^{2}} d x=\frac{1}{\pi} \int_{0}^{\infty} e^{-u} \int_{\mathbb{R}} e^{i \gamma x} e^{-u x^{2}} d x
$$

The formula (25) for the Poisson kernel can be obtained from the subordination identity,

$$
e^{-\tau|\xi|}=\frac{1}{\sqrt{\pi}} \int_{0}^{\infty} \frac{e^{-u}}{\sqrt{u}} e^{-\frac{(\tau|\xi|)^{2}}{4 u}} d u
$$


giving

$$
U(i \tau, x, y)=\frac{1}{\sqrt{\pi}} \int_{0}^{\infty} \int_{\mathbb{R}^{n}} \frac{e^{-u}}{\sqrt{u}} e^{-\frac{(\tau|\xi|)^{2}}{4 u}} e^{i\langle x-y, \xi\rangle} d \xi d u .
$$

Interchanging the order of integration then gives,

$$
U(i \tau, x, y)=\frac{1}{\sqrt{\pi}} \int_{0}^{\infty} \frac{e^{-u}}{\sqrt{u}}\left(\int_{T_{x} M} e^{-\frac{(\tau|\xi|)^{2}}{4 u}} e^{i\langle x-y, \xi\rangle} d \xi\right) d u .
$$

Substituting the formula for the heat kernel on $\mathbb{R}^{n}$,

$$
e^{-t \Delta}=(4 \pi t)^{-n / 2} e^{-\frac{|x-y|^{2}}{4 t}},
$$

we get

$$
\begin{aligned}
e^{-t \sqrt{\Delta}} & =\frac{t}{2 \sqrt{\pi}} \int_{0}^{\infty} e^{-\frac{t^{2}}{4 u}} e^{-u \Delta} u^{-3 / 2} d u \\
& =\frac{t}{2 \sqrt{\pi}} \int_{0}^{\infty} e^{-\frac{t^{2}}{4 u}}(4 \pi u)^{-n / 2} e^{-\frac{|x-y|^{2}}{4 u}} u^{-3 / 2} d u \\
& =C_{n} \tau \int_{0}^{\infty} e^{-\theta\left(\tau^{2}+|x-y|^{2}\right)} \theta^{(n-1) / 2} d \theta=C_{n} \tau\left(\tau^{2}+|x-y|^{2}\right)^{-\frac{n+1}{2}} .
\end{aligned}
$$

In the last step we put $\theta=\frac{1}{4 u}$.

3.7. Wave kernels and Poisson kernels on spaces of constant curvature. As further illustrations, we consider the Poisson-wave kernels on spaces of non-zero constant curvature following $[\mathrm{T}]$.

3.7.1. The sphere $\mathbb{S}^{n}$. Let $A=\sqrt{\Delta+\left(\frac{n-1}{4}\right)^{2}}$. Then the Poisson operator $e^{-t A}$ is given by

$$
\begin{aligned}
U\left(i \tau, \omega, \omega^{\prime}\right) & =C_{n} \frac{\sinh t}{\left(\cosh \tau-\cos r\left(\omega, \omega^{\prime}\right)\right)^{\frac{n+1}{2}}} \\
& =C_{n} \frac{\partial}{\partial \tau}\left(\cosh \tau-\cos r\left(\omega, \omega^{\prime}\right)\right)^{-\frac{n-1}{2}} .
\end{aligned}
$$

Here, $r\left(\omega, \omega^{\prime}\right)$ is the distance between points of $\mathbb{S}^{n}$. This formula is proved in [T] using the Poisson integral formula for a ball. Note that the addition of $\frac{n-1}{4}$ simplifies the formula and makes the operator strictly positive.

The wave kernel is the boundary value:

$$
e^{i t A}(x, y)=U(t, x, y)=C_{n} \sin t\left(\cos (t+i 0)-\cos r\left(\omega, \omega^{\prime}\right)^{-\frac{n+1}{2}} .\right.
$$

We see the kernel structure emphasized in Remark 3.5.

The analytically continued Poisson kernel is

$$
e^{(-\tau+i t) A}(\zeta, \omega)=C_{n} \sinh t(\cosh (\tau+i t)-\cos r(\omega, \zeta))^{-\frac{n+1}{2}} .
$$

It is singular on the complex characteristic conoid

$$
\cosh (\tau+i t)-\cosh r\left(\zeta, \bar{\zeta}^{\prime}\right)=0
$$


3.7.2. Wave kernel on Hyperbolic space. On hyperbolic space we define $A=\sqrt{\Delta-\left(\frac{n-1}{2}\right)^{2}}$, which brings the continuous spectrum down to zero. The wave kernel on hyperbolic space is obtained [T] by analytic continuation of the wave kernel of $\frac{\sin t A}{A}$ on the sphere by changing $r \rightarrow i r:$

$$
\lim _{\varepsilon \rightarrow 0^{+}}-2 C_{n} \operatorname{Im}(\cos (i t-\varepsilon)-\cosh r)_{+}^{-\frac{n-1}{2}}
$$

It follows that the Poisson kernel is

$$
U(i \tau, x, y)=\sinh t(\cosh (t+i 0)-\cosh r)^{-\frac{n+1}{2}} .
$$

We again see the kernel structure emphasized in Remark 3.5. The Poisson kernel and wave kernels for $e^{i t \sqrt{\Delta}}$ rather than $e^{i t A}$ are derived in [JL] for hyperbolic quotients using the subordination method (\$3.6) and the heat kernel. The use of $\sqrt{\Delta}$ rather than $A$ leads to lower order terms.

There is an alternative approach using Fourier analysis on hyperbolic space, where the exponential functions $e^{(i \lambda+1)\langle z, b\rangle}$ play the role of plane waves. Here, $z$ is in the interior of hyperbolic space and $b$ lies on its boundary and $\langle z, b\rangle$ is the distance of the horosphere through $z, b$ to 0 . For background we refer to [Hel]. We have,

$$
e^{i t A}(x, y)=\int_{0}^{\infty} e^{i t \lambda}\left\{\int_{B} e^{(i \lambda+1)\langle z, b\rangle} e^{(-i \lambda+1)\langle w, b\rangle} d b\right\} d p(\lambda),
$$

where $d p(\lambda)$ is the Planchere measure and $d b$ is the standard measure on the boundary (a sphere). This formula is the analogue of (24). The inner integral over $B$ is a spherical function $\varphi_{\lambda}(r(z, w))$ and is the hyperbolic analogue of a Bessel function. The analytic continuation of the Poisson kernel

$$
e^{-\tau A}(x, y)=\int_{0}^{\infty} e^{-\tau \lambda}\left\{\int_{B} e^{(i \lambda+1)\langle z, b\rangle} e^{(-i \lambda+1)\langle w, b\rangle} d b\right\} d p(\lambda)
$$

can be easily read off from this expression.

\section{The Hadamard-Feynman fundamental solution and Hadamard'S PARAMETRIX}

In his seminal work [H], Hadamard constructed a solution of $\square E=0$ for $t>0$ which has the singularity $\Gamma^{\frac{-m+2}{2}}, \quad m=n+1=\operatorname{dim} M \times \mathbb{R}$ (recall that $\Gamma$ is defined by (13)) . Note the analogy to the elliptic case where the Green's function (the kernel of $\Delta^{-1}$ ) has the singularity $r^{-n+2}$ if $n>2$.

The fundamental solution is more complicated in even spacetime dimensions (i.e. odd space dimensions). Hadamard found the general solution as follows:

- The elementary solution in odd spacetime dimensions has the form

$$
U \Gamma^{-\frac{m-2}{2}}
$$

where

$$
U=U_{0}+\Gamma U_{1}+\cdots+\Gamma^{h} U_{h}+\cdots
$$

is a holomorphic function. (This $U$ is not the half-wave propagator!). 
- The elementary solution in even spacetime dimensions has the form

$$
U \Gamma^{-\frac{m-2}{2}}+V \log \Gamma+W
$$

where

$$
U=\sum_{j=0}^{m-1} U_{j} \Gamma^{j}, \quad V=\sum_{j=0}^{\infty} V_{j} \Gamma^{j}, \quad W=\sum_{j=1}^{\infty} W_{j} \Gamma^{j} .
$$

Hadamard's formulae for the fundamental solutions pre-date the Schwartz theory of distributions. We follow his approach of describing the fundamental solutions as branched meromorphic functions (possibly logarithmically branched) on complexified spacetime. In modern terms $\Gamma^{\alpha}($ resp. $\log \Gamma)$ would be defined as the distributions $(\Gamma+i 0)^{\alpha}(\operatorname{resp} \cdot \log (\Gamma+i 0))$ as in the constant curvature cases. Hadamard implicitly worked in the complexified setting. For background on $\log (x+i 0)$, see [GeSh] Ch. III.4.4.

TheOREM 4.1. (Hadamard, 1920) With the $U_{j}, V_{k}, W_{\ell}$ defined as above,

- In odd spacetime dimensions, there exists a formal series $U$ as above so that $E=$ $U \Gamma^{\frac{2-m}{2}}$ solves $\square E=\delta_{0}(t) \delta_{y}(x)$. If $(M, g)$ is real analytic, the series $U=\sum_{j=0}^{\infty} U_{j} \Gamma^{j}$ converges absolutely for $|\Gamma|<\varepsilon$ sufficient small, i.e. near the characteristic conoid and admits a holomorphic continuation to a complex neighborhood of $\mathcal{C}_{\mathbb{C}}$.

- In even spacetime dimensions, $E=U \Gamma^{\frac{2-m}{2}}+V \log \Gamma+W$ solves $\square E=\delta_{0}(t) \delta_{y}(x)$. If $(M, g)$ is real analytic, all of the series for $U, V, W$ converge for $|\Gamma|$ small enough and admit analytic continuations to a neighborhood of $\mathcal{C}_{\mathbb{C}}$

In the smooth case, the series do not converge. But if they are truncated at some $j_{0}$, the partial sum defines a parametrix, i.e. a fundamental solution modulo functioins in $C^{j_{0}}$. By the Levi sums method (Duhamel principle) the parametrix differs from a true fundamental solution by a $C^{j_{0}}$ kernels. We are mainly interested in real analytic $(M, g)$ in this article and do not go into details on the last point. We note that the singularities of the kernel are due to the factors $\Gamma^{\frac{2-m}{2}}, \log \Gamma$, which are branched meromorphic (and logarithmic) kernels. The terms are explicitly evaluated in the case of hyperbolic quotients in [JL. We also refer to Chapter 5.2 of Gar for a somewhat modern presentation of the proof.

It may be of interest to note that this construction only occupies a third of Hadamard's book $[\mathrm{H}]$. The rest is devoted to the use of such kernels to solve the Cauchy problem, using Green's formula applied to a domain obtained by intersecting the backward characteristic conoid from a point $(t, x)$ of spacetime with the Cauchy hypersurface. The integrals over the lightlike (null) part of the boundary caused serious trouble since the factors $\Gamma^{\frac{2-m}{2}}$ are infinite along them and need to be re-normalized. This was the origin of Hadamard's finite parts of divergent integrals. Riesz used analytic continuation methods instead to define the forward fundamental solution in $[\mathrm{R}]$.

4.1. Sketch of proof of Hadamard's construction. Let $\Theta=\sqrt{\operatorname{det}\left(g_{j k}\right)}$ be the volume density in normal coordinates based at $y, d V=\Theta(y, x) d x$. That is,

$$
\Theta(x, y)=\left|\operatorname{det} D_{\exp _{x}^{-1}(y)} \exp _{x}\right| .
$$

Fix $x \in M$ and endow $B_{\varepsilon}(x)$ with geodesic polar coordinates $r, \theta$. That is, use the chart $\exp _{x}^{-1}: B_{r}(x) \rightarrow B_{x, r}^{*} M$ combined with polar coordinates on $T_{x}^{*} M$. Then $g^{11}=1, g^{1 j}=0$ for 
$j=2, \ldots, n$. Also, $d V=\Theta(x, y) d y=\Theta(x, r, \theta) r^{n-1} d r d \theta$. So the volume density $J$ relative to Lebesgue measure $d r d \theta$ in polar coordinates is given by $J=r^{n-1} \Theta$.

In these coordinates,

$$
\Delta=\frac{1}{J} \sum_{j, k=1}^{n} \frac{\partial}{\partial x_{j}}\left(J g^{j k} \frac{\partial}{\partial x_{k}} \cdot\right)=\frac{\partial^{2}}{\partial r^{2}}+\frac{J^{\prime}}{J} \frac{\partial}{\partial r}+L,
$$

where $L$ involves no $\frac{\partial}{\partial r}$ derivatives. Equivalently,

$$
\Delta=\frac{\partial^{2}}{\partial r^{2}}+\left(\frac{\Theta^{\prime}}{\Theta}+\frac{n-1}{r}\right) \frac{\partial}{\partial r}+L
$$

The first step in the parametrix construction is to find the phase function. Hadamard chooses to use $\Gamma(\underline{13})$. In the Lortenzian metric, $\Gamma$ satisfies

$$
\nabla \Gamma \cdot \nabla \Gamma=4 \Gamma \text {. }
$$

This is not the standard Eikonal equation $\sigma_{\square}(d \varphi)=0$ of geometric optics, but rather has the form

$$
\sigma_{\square}(d \Gamma)=4 \Gamma .
$$

But $\Gamma$ is a good phase, since the Lagrangian submanifold

$$
\left\{\left(t, d_{t} \Gamma, x, d_{x} \Gamma, y,-d_{y} \Gamma\right)\right\}
$$

is the graph of the bichafacteristic flow. This is because the $d_{x} r(x, y)$ is the unit vector pointing along the geodesic joining $x$ to $y$ and $d_{y} r(x, y)$ is the unit vector pointing along the geodesic pointing from $y$ to $x$.

To proceed, we introduce the simplifying notation

$$
M=\square \Gamma=-4-2 r \frac{(n-1)}{r}-2 r \frac{\Theta_{r}}{\Theta}=2 m+2 r \frac{\Theta_{r}}{\Theta}
$$

where $m=n+1$. We then have,

$$
\begin{aligned}
\square\left[f(\Gamma) U_{j}\right] & =\square[f(\Gamma)] U_{j}+2 \nabla[f(\Gamma)] \nabla U_{j}+f(\Gamma) \square U_{j} \\
& =\left(f^{\prime \prime}(\Gamma) \nabla(\Gamma) \cdot \nabla(\Gamma)+f^{\prime}(\Gamma) \square(\Gamma)\right) U_{j}+2 f^{\prime}(\Gamma) \nabla \Gamma \cdot \nabla U_{j}+f(\Gamma) \square U_{j} .
\end{aligned}
$$

In addition to (28), we further have

$$
\left\{\begin{array}{l}
\square \Gamma=4+\frac{J_{r}}{J} 2 r \\
\nabla \Gamma \cdot \nabla=\nabla\left(t^{2}-r^{2}\right) \cdot \nabla=2\left(t \frac{\partial}{\partial t}+r \frac{\partial}{\partial r}\right)=2 s \frac{d}{d s},
\end{array}\right.
$$

where we recall that that we are using the Lorentz metric of signature +--- . Here $s^{2}=\Gamma$, and the notation $s \frac{d}{d s}$ refers to differentiation along a spacetime geodesic.

We then have

$$
\left.\square\left[f(\Gamma) U_{j}\right]=\left(f^{\prime \prime}(\Gamma)(4 \Gamma)+f^{\prime}(\Gamma)\left(4+\frac{J_{r}}{J} 2 r\right)\right)\right) U_{j}+2 f^{\prime}(\Gamma)\left(-2 s \frac{d}{d s} U_{j}\right)+f(\Gamma) \square U_{j} .
$$

We now apply this equation in the cases $f=x^{\frac{2-m}{2}+j}$. (and later to $f=\log x$ ), in which case

$$
f^{\prime}=\left(\frac{2-m}{2}+j\right) x^{\frac{2-m}{2}+j-1}, \quad f^{\prime \prime}=\left(\frac{2-m}{2}+j\right)\left(\frac{2-m}{2}+j-1\right) x^{\frac{2-m}{2}+j-2} .
$$


We then attempt to solve

$$
\square\left(\Gamma^{\frac{2-m}{2}} \sum_{j=0}^{\infty} U_{j} \Gamma^{j}\right)=0
$$

away from the characteristic conoid by seting the coefficient of each power $\Gamma^{\frac{2-m}{2}+j-1}$ of $\Gamma$ equal to zero. The resulting 'transport equations' are

$$
\begin{aligned}
& {\left[-4\left(\left(\frac{2-m}{2}+j\right)\left(\frac{2-m}{2}+j-1\right)\right)+\left(\frac{2-m}{2}+j\right)\left(-4-\frac{J_{r}}{J} 2 r\right)\right)} \\
& +2\left(\left(\frac{2-m}{2}+j\right)\left(-2 s \frac{d}{d s}\right]\right) U_{j}+\square U_{j-1}=0 .
\end{aligned}
$$

They are impossible to solve for all $j$ when $m$ is even because the common factor $\left(\frac{2-m}{2}+j\right)$ vanishes when $j=\frac{m-2}{2}$. We thus first assume that $m$ is odd so that it is non-zero for all $j$ We then recursively solve Hadamard's transport equations in even space dimensions,

$$
4 s \frac{d U_{k}}{d s}+\left(M-2 m+2 r \frac{J_{r}}{J}\right) U_{k}=-\square U_{k-1} .
$$

When $k=0$ we get

$$
2 s \frac{d U_{0}}{d s}+2 s \frac{\Theta_{s}}{\Theta}=0
$$

which is solved by

$$
U_{0}=\Theta^{-\frac{1}{2}}
$$

The solution of the $\ell$ th transport equation is then,

$$
U_{\ell}=-\frac{U_{0}}{4 \ell s^{m+\ell}} \int_{0}^{s} U_{0}^{-1} s^{\ell+m-1} \square U_{\ell-1} d s .
$$

Hence we have a formal solution with the singularity of the Green's function in the elliptic case, and by comparison with the Euclidean case we see that it solves $\square E=\delta_{0}$.

We now consider the necessary modifications in the case of even dimensional spacetimes. In this case, $\Gamma^{\frac{2-m}{2}} \Gamma^{j}$ is always an integer power. If we could solve the transport equation for $j=\frac{m-2}{2}$, the resulting term would be regular with power $\Gamma^{0}$. The problem is that $\Gamma^{0}$ should actually be a term with a logarithmic singularity $\log \Gamma$.

Thus the parametrix (29) is inadequate in even spacetime dimensions. Hadamard therefore introduced a logarithmic term $V \log (\Gamma)$. By a similar calculation to the above,

$$
\square[(\log \Gamma) V]=\left(-\Gamma^{-2}(4 \Gamma)+\Gamma^{-1}\left(-4-\frac{J_{r}}{J} 2 r\right)\right) V+2 \Gamma^{-1}\left(-2 s \frac{d}{d s} V\right)+\log \Gamma \square V .
$$

Due to (28), all terms except the logarithmic term have the same singularity $\Gamma^{-1}$. On the other hand, the only way to eliminate the logarithmic term is to insist that $\square V=0$. We further assume that

$$
V=\sum_{j=0}^{\infty} V_{j} \Gamma^{j} .
$$

We then return to the unsolvable transport equations for $U_{j}$ for $j \geq \frac{m-2}{2}$, which now acquires the new $V_{0}$ term to become:

$$
\begin{aligned}
& {\left[-4\left(\left(\frac{2-m}{2}+j\right)\left(\frac{2-m}{2}+j-1\right)\right)+\left(\frac{2-m}{2}+j\right)\left(-4-\frac{J_{r}}{J} 2 r\right)\right)+2\left(\left(\frac{2-m}{2}+j\right)\left(-2 s \frac{d}{d s}\right]\right) U_{j}+\square U_{j-1}} \\
& +\Gamma^{-1}\left[\left(4+\left(-4-\frac{J_{r}}{J} 2 r\right)\right)+2 \frac{d}{d s}\right] V_{0}=0 .
\end{aligned}
$$


When $j=\frac{m-2}{2}$, everything cancels in the $\Gamma^{-1}$ term except $\square U_{m-1}$. Hence, we drop the $U_{j}$ for $j \geq \frac{m-2}{2}$ and assume the non logarithmic part is just the finite sum $\sum_{j=0}^{m-1} U_{j} \Gamma^{j}$. But adding in the $V_{0}$ term we get the transport equation,

$$
-4 s \frac{d V_{0}}{d s}-2 r \frac{J_{r}}{J} V_{0}=-\square U_{m-1} .
$$

Here, $U_{m-1}$ is known and we solve for $V_{0}$ to get,

$$
V_{0}=-\frac{U_{0}}{4 s^{m}} \int_{0}^{s} U_{0}^{-1} s^{m-1} \square U_{m-1} d s .
$$

The condition $\square V=0$ imposed above then determines the rest of the coefficients $V_{j}$,

$$
V_{\ell}=-\frac{U_{0}}{4 \ell s^{m+\ell}} \int_{0}^{s} U_{0}^{-1} s^{\ell+m-1} \square V_{\ell-1} d s .
$$

We now have two equations: the original $\square\left(U \Gamma^{\frac{2-m}{2}} U+V \log \Gamma\right)=0$ and the new $\square V=0$. By solving the transport equations for $U_{0}, \ldots, U_{m-1}, V_{0}, V_{j}(j \geq 1)$ we obtain a solution of an inhomogeneous equation of the form,

$$
\square\left(U \Gamma^{\frac{2-m}{2}}+V \log \Gamma\right)=\sum_{j=0} w_{j} \Gamma^{j},
$$

where the right side is regular. To complete the construction, we add a new term of the form $W=\sum_{\ell=1}^{\infty} W_{\ell}\left(r^{2}-t^{2}\right)^{\ell}$ in order to ensure that

$$
\left.\square \sum_{j=0}^{m-1} U_{j}\left(r^{2}-t^{2}\right)^{-m+j}+V \log \left(r^{2}-t^{2}\right)+W\right)=0
$$

away from the characteristic conoid. It then suffices to find $W_{j}$ so that

$$
\square \sum_{j=1}^{\infty} W_{j} \Gamma^{j}=\sum_{j=0} w_{j} \Gamma^{j} .
$$

This leads to more transport equations which are always solvable (by the Cauchy-Kowalevskaya theorem). This concludes the sketch of the proof of Theorem 4.1.

4.2. Convergence in the real analytic case. The above parametrix construction was formal. However, when the metric is real analytic, Hadamard proved that the formal series converges for $|t|$ and $|\Gamma|$ sufficiently small. The convergence proof based on the method of majorants.

Theorem 4.2. [H] (see also [Gar]) Assume that $(M, g)$ is real analytic. Then there exists $K>0$ so that the Hadamard parametrix converges for any $(t, y)$ such that $t \neq 0, r(x, y)<$ $\varepsilon=\operatorname{inj}\left(x_{0}\right)$ and

$$
\left|t^{2}-r^{2}\right| \leq \frac{\left(\left(1-\frac{\|y\|}{\varepsilon}\right)^{2}\right.}{\left(1+\frac{m_{1}}{\varepsilon}+\frac{m_{1}^{2}}{\varepsilon^{2}}\right) K}, \quad\left(m_{1}=\frac{m-2}{2}\right) .
$$


It follows that the Hadamard fundamental solutions holomorphically extend to a neighborhood of $\mathcal{C}_{\mathbb{C}}$ as branched meromorphic functions iwith $\mathcal{C}_{\mathbb{C}}$ as branch locus. To obtain single valued distributions, one then needs to restrict the kernels to regions where a unique branch can be defined.

4.3. Away from $\mathcal{C}_{\mathbb{R}}$. A further complication is that the fundamental solution has only been constructed in a neighborhood of $\mathcal{C}_{\mathbb{R}}$. But it is known to be real analytic on $(\mathbb{R} \times M \times M) \backslash \mathcal{C}_{\mathbb{R}}$ and that it extends to a holomorphic kernel away from a neighborhood of $\mathcal{C}_{\mathbb{C}}$. To prove this it suffices to note that the analytic wave front set of the fundamental solution is $\mathcal{C}_{\mathbb{R}}$. A more detailed proof is given by Mizohata [Miz, Miz2 for 'elementary solutions', i.e. solutions $E(t, x, y)$ of $\square E=0$ such as $\cos t \sqrt{\Delta}$ or $\frac{\sin t \sqrt{\Delta}}{\sqrt{\Delta}}$ whose Cauchy data is either zero or a delta function. Here, $\square$ operates in the $x$ variable with $y$ as a parameter. To analyze the wave kernels away from the characteristic conoid, Mizohata makes the decomposition

$$
E(t, x, y)=E_{N}(t, x, y)+w_{N}(t, x, y)+z_{N}(t, x, y)
$$

where

- $\square E_{N}=f_{N}$ with $f_{N} \in C^{N-1}(\mathbb{R} \times M \times M)$. Also, $\left.E_{N}(t, x, y)\right|_{t=0}=\delta_{y}+a(x, y)$ with $a(x, y) \in C^{\omega}(M \times M)$ (in fact it is independent of $y$ );

- $\square w_{N}(t, x, y)=-f_{N}(t, x, y)$, with $w(0, x, y)=0$;

- $\square z_{N}=0, z_{N}(0, x, y)=-a(x, y)$.

The same kind of decomposition applies to the Hadamard fundamental solution. The term constructed by the parametrix method is $E_{N}$. By solving the above equations, it is shown in Miz that the sum is analytic away from $\mathcal{C}_{\mathbb{R}}$. One can see that the Hadamard method is only a branched Laurent type expansion near $\mathcal{C}_{\mathbb{R}}$ by considering the examples in $\$ 3.7$ of the kernels for spaces of constant curvature.

\section{Hadamard PARAmetrix FOR the Poisson-WAVE KeRnel}

We are most interested in the Hadamard parametrix for the half-wave kernel, which does not seem to have been discussed in the literature. We are more generally interested in the Poisson-wave semi-group $e^{i(t+i \tau) \sqrt{\Delta}}$ for $\tau>0$. The Poisson-wave lernel

$$
U(t+i \tau, x, y)=\sum_{j} e^{\left(i(t+i \tau) \lambda_{j}\right.} \varphi_{j}(x) \varphi_{j}(y)
$$

is a real analytic kernel which possesses an analytic extension to a Grauert tube. Thus, there exists a non-zero analytic radius $\tau_{a n}>0$ so that the Poisson kernel admits a holomorphic extension $U(t+i \tau, \zeta, y)$ to $M_{\tau} \times M$ for $\tau \leq \tau_{a n}$. Since

$$
U(i \tau) \varphi_{\lambda}=e^{-\tau \lambda} \varphi_{\lambda}^{\mathbb{C}},
$$

the eigenfunctions analytically extend to the same maximal tube as does $U(i \tau)$.

We would like to construct a Hadamard type parametrix for (32). We may derive it from the Feynman-Hadamard fundamental solution in Proposition 3.2 using that

$$
\frac{d}{d t} \frac{e^{i|t| \sqrt{\Delta}}}{\sqrt{\Delta}}=i \operatorname{sgn}(t) e^{i|t| \sqrt{\Delta}}
$$


and

$$
e^{i t \sqrt{\Delta}}=\frac{1}{i} H(t) \frac{d}{d t} \frac{e^{i|t| \sqrt{\Delta}}}{\sqrt{\Delta}}-\frac{1}{i} H(-t) \frac{d}{d t} \frac{e^{-i|t| \sqrt{\Delta}}}{\sqrt{\Delta}} .
$$

Hence,

$$
\frac{d}{i d t} U_{F}(t)=e^{i t \sqrt{\Delta}}, \quad(t>0)
$$

The restriction to $t>0$ is consistent with the kernel analysis in Remark 3.5, reflecting the fact that $e^{i t \sqrt{\Delta}}(x, y)$ has the singularity $\left((t+i 0)^{2}-r^{2}\right)^{\frac{-m}{2}}$ (in odd spacetime dimensions) while $U_{F}(t)$ has the singularity $\left(t^{2}-r^{2}+i 0\right)^{\frac{2-m}{2}}$. We note (again) that $\left((t+i 0)^{2}-r^{2}\right)^{\alpha}=\left(t^{2}-r^{2}+i 0\right)^{\alpha}$ for $t>0$.

From Theorem 4.1 we conclude:

Corollary 5.1. Let $(M, g)$ be real analytic. Then with the $U_{j}, V_{k}, W_{\ell}$ defined as in Theorem 4.1, we have:

- In odd spacetime dimensions, for $t>0$ the Poisson-wave kernel $U(t+i \tau, x, y)(\tau>$ $0)$ has the form $A \Gamma^{\frac{-m}{2}}$ where $A=\sum_{j=0}^{\infty} A_{j} \Gamma^{j}$ with $A_{j}$ holomorphic. The series converges absolutely to a holomorphic function for $|\Gamma|<\varepsilon$ sufficient small, i.e. near the characteristic conoid.

- In even spacetime dimensions, for $t>0$, the Poisson-wave kernel has the form $B \Gamma^{\frac{-m}{2}}+C \log \Gamma+D$ where the coefficients $B, C, D$ are holomorphic in a neighborhood of $\mathcal{C}_{\mathbb{C}}$, and have the same $\Gamma$ expansions as $A$.

We use this parametrix to prove Theorem 2 (1).

5.1. Hadamard parametrix as an oscillatory integral with complex phase. Corollary 5.1 gives a precise description of the singularities of the Poisson-wave propagator. It implicitly describes the kernel as a Fourier integral kernel. We now make this description explicit in the real domain. In the following sections, we extend the description to the complex domain.

We first express $\Gamma^{\frac{-m}{2}+j}$ as an oscillatory integral with one phase variable using the wellknown identity

$$
\int_{0}^{\infty} e^{i \theta \sigma} \theta_{+}^{\lambda} d \lambda=i e^{i \lambda \pi / 2} \Gamma(\lambda+1)(\sigma+i 0)^{-\lambda-1}
$$

At least formally, this leads to the representation

$$
\int_{0}^{\infty} e^{i \theta\left(t^{2}-r^{2}\right)} \theta_{+}^{\frac{n-1}{2}-j} d \theta=i e^{i\left(\frac{n-1}{2}-j\right) \pi / 2} \Gamma\left(\frac{n-1}{2}-j+1\right)\left(t^{2}-r^{2}+i 0\right)^{j-\frac{n-1}{2}-1}
$$

for the principal term of the Poisson-wave. Here, the notation $\Gamma=t^{2}-r^{2}$ unfortunately clashes with that for the Gamma function, and we temporarily write out its defintion.

In even space dimensions, the Hadamard parametrix for the Hadamard-Feynman fundamental solution thus has the form

$$
\sum_{j=0}^{\infty} U_{j}(t, x, y) \Gamma^{\frac{1-n}{2}+j}=\int_{0}^{\infty} e^{i \theta\left(t^{2}-r^{2}\right)}\left(\sum_{j=0}^{\infty} U_{j}(t, x, y)\left(i e^{i\left(\frac{n-1}{2}-j\right) \pi / 2}\right)^{-1} \frac{\theta_{+}^{\frac{n-3}{2}-j}}{\Gamma\left(\frac{n-3}{2}-j+1\right)}\right) d \theta .
$$


Here we follow Hadamard's notation, but it is simpler to re-define the coefficients $U_{j}$ so that the $\Gamma$-factors appear on the left side as in [Be] (7). We thus define

$$
\mathcal{U}_{j}(t, x, y)=\left(\left(i e^{i\left(\frac{n-1}{2}-j\right) \pi / 2}\right)^{-1} \frac{1}{\Gamma\left(\frac{n-3}{2}-j+1\right)}\right) U_{j}(t, x, y) .
$$

By the duplication formula $\Gamma(z) \Gamma(1-z)=\frac{\pi}{\sin \pi z}$ with $z=\frac{m}{2}-k-\frac{\alpha}{2}$, i.e.

$$
\Gamma\left(\frac{m}{2}-j-\frac{\alpha}{2}\right)=(-1)^{j} \frac{\pi}{\sin \pi\left(\frac{m}{2}-\frac{\alpha}{2}\right)} \frac{1}{\Gamma\left(-\frac{m}{2}+1+j+\frac{\alpha}{2}\right)},
$$

it follows that

$$
U_{j}(t, x, y)=\left((-1)^{j} \frac{\pi}{\sin \pi\left(\frac{m}{2}-\frac{\alpha}{2}\right)} \frac{1}{\Gamma\left(-\frac{m}{2}+1+j+\frac{\alpha}{2}\right)}\right) \mathcal{U}_{j}(t, x, y),
$$

so that the formula in odd spacetime dimensions becomes

$$
\begin{aligned}
C_{n} \frac{1}{\sin \pi\left(\frac{m}{2}-\frac{\alpha}{2}\right)} \sum_{j=0}^{\infty}(-1)^{j} \frac{\mathcal{U}_{j}(t, x, y)}{\Gamma\left(-\frac{m}{2}+1+j+\frac{\alpha}{2}\right)}\left(t^{2}-r^{2}\right)^{-\frac{m-2}{2}+j} & \\
& =\int_{0}^{\infty} e^{i \theta\left(t^{2}-r^{2}\right)}\left(\sum_{j=0}^{\infty} \mathcal{U}_{j}(t, x, y) \theta_{+}^{\frac{n-3}{2}-j}\right) d \theta .
\end{aligned}
$$

The amplitude in the right side of (39) is then a formal analytic symbol,

$$
A(t, x, y, \theta)=\sum_{j=0}^{\infty} \mathcal{U}_{j}(t, x, y) \theta_{+}^{\frac{n-3}{2}-j},
$$

Due to the Gamma-factors appearing in the identity (37), convergence of the series on the left side of (39) does not imply convergence of the series (40). However, there exists a realization of the formal symbol (40) by a holmorphic symbol

$$
\mathcal{A}(t, x, y, \theta)=\sum_{0 \leq j \leq \frac{\theta}{e C}} \mathcal{U}_{j}(t, x, y) \theta_{+}^{\frac{n-3}{2}-j}
$$

and one obtains an analytic parametrix

$$
U(t, x, y)=\int_{0}^{\infty} e^{i \theta \Gamma} \mathcal{A}(t, x, y, \theta) d \theta
$$

which approximates the wave kernel for small $|t|$ and $(x, y)$ near the diagonal up to a holomorphic error, whose amplitude is exponentially decaying in $\theta$. Here, we recall (see [Sj], p. 3 and section 9) that a classical formal analytic symbol ([Sj], page 3) on a domain $\Omega \subset \mathbb{C}^{n}$ is a formal semi-classical series

$$
a(z, \lambda)=\sum_{k=0}^{\infty} a_{k}(z) \lambda^{-k}
$$

where $a_{k}(z, \lambda) \in \mathcal{O}(\Omega)$ for all $\lambda>0$. Then for some $C>0$, the $a_{k}(z) \in \mathcal{O}(\Omega)$ satisfy

$$
\left|a_{k}(z)\right| \leq C^{k+1} k^{k}, \quad k=0,1,2, \ldots
$$


A realization of the formal symbol is a genuine holomorphic symbol of the form,

$$
a(z, \lambda)=\sum_{0 \leq k \leq \frac{\lambda}{e C}} a_{k}(z) \lambda^{-k}
$$

It is an analytic symbol since, with the index restriction,

$$
\left|a_{k}(z) \lambda^{-k}\right| \leq C_{\Omega}\left(\frac{C k}{\lambda}\right)^{k} \leq C e^{-k}
$$

Hence the series converges uniformly on $\Omega$ to a holomorphic function of $z$ for each $\lambda$.

Returning to (40), the Hadamard-Riesz coefficients $\mathcal{U}_{j}$ are determined inductively by the transport equations

$$
\left\{\begin{array}{l}
\frac{\Theta^{\prime}}{2 \Theta} \mathcal{U}_{0}+\frac{\partial \mathcal{U}_{0}}{\partial r}=0 \\
\operatorname{4ir}(x, y)\left\{\left(\frac{k+1}{r(x, y)}+\frac{\Theta^{\prime}}{2 \Theta}\right) \mathcal{U}_{j+1}+\frac{\partial \mathcal{U}_{j+1}}{\partial r}\right\}=\Delta_{y} \mathcal{U}_{j}
\end{array}\right.
$$

whose solutions are given by:

$$
\left\{\begin{array}{l}
\mathcal{U}_{0}(x, y)=\Theta^{-\frac{1}{2}}(x, y) \\
\mathcal{U}_{j+1}(x, y)=\Theta^{-\frac{1}{2}}(x, y) \int_{0}^{1} s^{k} \Theta\left(x, x_{s}\right)^{\frac{1}{2}} \Delta_{2} \mathcal{U}_{j}\left(x, x_{s}\right) d s
\end{array}\right.
$$

where $x_{s}$ is the geodesic from $x$ to $y$ parametrized proportionately to arc-length and where $\Delta_{2}$ operates in the second variable.

As discussed above, the representation (39) does not suffice when $n$ is odd, since $\Gamma(z)$ and $\theta_{+}^{z}$ have poles at the negative integers. To rescue the representation when $n$ is odd, we need to use the distributions $\theta_{+}^{-n}$ with $n=1,2, \ldots$, defined as follows (see [Ho] Vol. I):

$$
\theta_{+}^{-k}(\varphi)=\int_{0}^{\infty}(\log \theta) \varphi^{(k)}(\theta) d x /(k-1) !+\varphi^{(k-1)}(0)\left(\sum_{j=1}^{k} 1 / j\right) /(k-1) ! .
$$

This family behaves in an unusal way under derivation,

$$
\frac{d}{d \theta} \theta_{+}^{-k}=-k \theta_{+}^{-k-1}+(-1)^{k} \delta_{0}^{(k)} / k !
$$

(see [Ho] Vol. I (3.2.2)") and is therefore sometimes avoided in the Hadamard-Riesz parametrix construction (as in $[\mathrm{Be}]$ ).

However, we have already constructed the parametrices and only want to express them in terms of the above oscillatory integrals to make contact with Fourier integral operator theory. In odd space dimensions, the Hadamard parametrices can be written in the form

$$
\begin{aligned}
& \int_{0}^{\infty} e^{i \theta \Gamma}\left(U_{0}(t, x, y) \theta_{+}^{m}+\cdots+U_{m} \theta_{+}^{0}\right) d \theta \\
& +\int_{0}^{\infty} e^{i \theta \Gamma}\left(U_{m+1} \theta_{+}^{-1}+U_{m+2} \theta_{+}^{-2}+\cdots\right) d \theta
\end{aligned}
$$

Again the amplitude is a formal symbol. To produce a genuine amplitude it needs to be replace by a realization which approximates it modulo a holomorphic symbol which is exponentially decaying in $\theta$.

We are paying close attention to the regularization of the integral at $\theta=0$, but only the behavior of the amplitude as $\theta \rightarrow \infty$ is relevant to the singularity. The terms with $\theta_{+}^{-k}$ for 
$k>0$ produce logarithmic terms in the kernel. If we use a smooth cutoff at $\theta=0$, we obtain distributions of the form

$$
u_{\mu}(\Gamma)=\int_{\mathbb{R}} e^{i \theta \Gamma} \chi(\theta) \theta^{\mu} d \theta
$$

where $\chi(\theta)=1$ for $\theta \geq 1$ and $\chi(\theta)=0$ for $\theta \leq \frac{1}{2}$. Then

$$
u_{-k}(\Gamma)=i^{k+1} \Gamma^{k-1} \log \Gamma, \quad \text { modulo } C^{\infty}, \quad u_{-k}(-\Gamma)=(-i)^{n+1} \Gamma^{n-1} \log \Gamma .
$$

Hence the terms with negative powers of $\theta_{+}$in (44) produce the logarithmic terms and the holomorphic terms.

Above, we discussed the Hadamard-Feynman fundamental solution, but for $t>0$ we only need to differentiate it in $t$ (according to Proposition 5.1 ) to obtain the parametrices for the Poisson-wave group. Away from the characteristic conoid the Schwartz kernels of the Poisson-wave group and Hadamard-Feynman fundamental solution are holomorphic by the theorem on propagation of analytic wave front sets [Ho, $\mathrm{Sj}$ ] (see also [Miz, Miz2]). The Fourier integral structure and mapping properties follow immediately from the order of the amplitude and from the exact formula for the phase.

5.2. Modified Hadamard parametrix with phase $t-r$ when $t \geq 0$. We now assume that $t \geq 0$ as well as $\tau>0$. We note that the phase $t^{2}-r^{2}$ for $U(t, x, y)$ factors as $(t-r)(t+r)$ with $t+r \geq 0$ when $t \geq 0$. Of course, $r(x, y) \geq 0$ in the real domain. We can therefore change variables $\theta \rightarrow(t+r) \theta$ in (41) to obtain the modified Hadamard parametrix,

$$
U(t, x, y)=(t+r)^{-1} \int_{0}^{\infty} e^{i \theta(t-r)} \mathcal{A}\left(t, x, y, \frac{\theta}{t+r}\right) d \theta, \quad(t \geq 0)
$$

with phase $t-r$. We note that it is singular when $r=0$ but we only intend to use it for

$r_{\mathbb{C}} \neq 0$. The amplitude $=(t+r)^{-1} \mathcal{A}\left(t, x, y, \frac{\theta}{t+r}\right)$ is a representative of an analytic symbol as long as $r+t \neq 0$ and $r \neq 0$.

\section{HÖRmAnder PARAmetrix FOR the POISSON-WAVE KERNEL}

A more familiar construction of $U(t, x, y)$ and its analytic continuation which is particularly useful for small $|t|$ is the one (24) based on the Fourier inversion formula. Its generalization to Riemannian manifolds is given by

$$
U(t, x, y)=\int_{T_{y}^{*} M} e^{i t|\xi|_{g_{y}}} e^{i\left\langle\xi, \exp _{y}^{-1}(x)\right\rangle} A(t, x, y, \xi) d \xi,
$$

for $(x, y)$ sufficiently close to the diagonal. We use this parametrix to prove Theorem 2 (2).

The amplitude is a polyhomogeous symbol of the form

$$
A(t, x, y, \xi) \sim \sum_{j=}^{\infty} A_{j}(t, x, y, \xi)
$$

where the asymptotics are in the sense of the symbol topology and where

$$
A_{j}(t, x, y, \tau \xi)=\tau^{-j} A_{j}(t, x, y, \xi), \quad \text { for }|\xi| \geq 1 .
$$

The principal term $A_{0}(t, x, y, \xi)$ equals 1 when $t=0$ on the diagonal, and the higher $A_{j}$ are determined by transport equations discussed in [DG]. 
It can be verified that in the case of real analytic $(M, g)$, the amplitude is a classical formal analytic symbol (see $\$ 5.1$ ). Hence if $\mathcal{A}(t, x, y, \xi)$ is a realization of the amplitude $A(t, x, y, \xi)$, then one obtains an analytic parametrix

$$
U(t, x, y)=\int_{T_{y}^{*} M} e^{i t|\xi|_{g_{y}}} e^{i\left\langle\xi, \exp _{y}^{-1}(x)\right\rangle} \mathcal{A}(t, x, y, \xi) d \xi
$$

which approximates the wave kernel for small $|t|$ and $(x, y)$ near the diagonal up to a holomorphic error, whose amplitude is exponentially decaying in $|\xi|$.

6.1. Subordination to the heat kernel. The parametrix (53) can also be obtained by subordinating the Poisson-wave kernel to the heat kernel in the sense of \$3.6. To make use of it, one needs to analytically the heat kernel to $M_{\tau}$. This analytic continuation was studied by Golse-Leichtnam-Stenzel in GLS., who proved the following: For any $x_{0} \in M$ there exists $\varepsilon, \rho>0$ and an open neighborhood $W$ of $x_{0}$ in $M_{\varepsilon}$ such that for $0<t<1$ and $(x, y) \in W \times W$,

where

$$
E(t, x, y)=N(t, x, y) e^{-\frac{r^{2}(x, y)}{4 t}}+R(t, x, y)
$$

$$
N(t, x, y)=\sum_{0 \leq j \leq \frac{1}{C t}} W_{j}(x, y) t^{j}
$$

as $t \downarrow 0^{+}$where $W_{j}(x, y)$ are the Hadamard-Minakshisundaram-Pleijel heat kernel coefficients. is an analytic symbol of ordern $n / 2$ with respect to $t^{-1}$ in the sense of [Sj]. As above, the remainder is exponentially small,

$$
|R(t, x, y)| \leq C e^{-\frac{\rho}{8 t}}
$$

with a uniform $C$ in $(x, y)$ as $t \downarrow 0^{+}$. The heat kernel itself obviously admits a holmoorphic extension in the open subset $\operatorname{Re} r_{\mathbb{C}}^{2}(x, y)>0$ ot $M_{\mathbb{C}} \times M_{\mathbb{C}}$.

\section{Complexified Poisson kernel as a COMPlex Fourier integral operator}

We now consider the Fourier integral operator aspects of the analytic continuation of the Poisson-wave kernel $U(t+i \tau, \zeta, y)$ for $\tau>0$ and $(\zeta, y) \in M_{\tau} \times M$, developing analogues of the results of $\$ 5.1$ in the complex domain. The aim is to prove that the analytically continued Poisson-wave kernel is a complex Fourier integral operator. We denote by $\mathcal{O}\left(M_{\varepsilon}\right)$ the space of holomorphic functions on the Grauert tube and by a slight abuse of notation we also denote by $\mathcal{O}\left(\partial M_{\varepsilon}\right)$ the $\mathrm{CR}$ holomorphic functions on the boundary $\partial M_{\varepsilon}$ of the strictly pseudo-convex domain $M_{\varepsilon}$ (the null space of the boundary Cauchy-Riemann operator $\bar{\partial}_{b}$.) In particular, we denote by $\mathcal{O}^{0}\left(\partial M_{\varepsilon}\right)=H^{2}\left(\partial M_{\varepsilon}\right)$ the Hardy space of boundary values of holomorphic functions of $M_{\varepsilon}$ which lie in $L^{2}\left(\partial M_{\varepsilon}\right)$ relative to the natural Liouville measure

$$
d \mu_{\tau}=(i \partial \bar{\partial} \sqrt{\rho})^{m-1} \wedge d^{c} \sqrt{\rho} .
$$

We further denote by $\mathcal{O}^{s+\frac{n-1}{4}}\left(\partial M_{\tau}\right)$ the Sobolev spaces of CR holomorphic functions on $\partial M_{\tau}$, i.e.

$$
\mathcal{O}^{s+\frac{m-1}{4}}\left(\partial M_{\tau}\right)=W^{s+\frac{m-1}{4}}\left(\partial M_{\tau}\right) \cap \mathcal{O}\left(\partial M_{\tau}\right)
$$

where $W_{s}$ is the $s$ th Sobolev space.

The spray

$$
\Sigma_{\tau}=\left\{\left(\zeta, r d^{c} \sqrt{\rho}(\zeta): r \in \mathbb{R}_{+}\right\} \subset T^{*}\left(\partial M_{\tau}\right)\right.
$$


of the contact form $d^{c} \sqrt{\rho}$ defines a symplectic cone. There exists a symplectic equivalence (cf. GS2])

$$
\iota_{\tau}: T^{*} M-0 \rightarrow \Sigma_{\tau}, \quad \iota_{\tau}(x, \xi)=\left(E\left(x, \tau \frac{\xi}{|\xi|}\right),|\xi| d^{c} \sqrt{\rho}_{E\left(x, \tau \frac{\xi}{|\xi|}\right)}\right) .
$$

The following theorem is stated in [Bou] (see also [Z3]):

TheOREM 7.1. (see [Bou, GS2, GLS]) For sufficiently small $\tau>0, U_{\mathbb{C}}(i \tau): L^{2}(M) \rightarrow$ $\mathcal{O}\left(\partial M_{\tau}\right)$ is a Fourier integral operator of order $-\frac{m-1}{4}$ with complex phase associated to the canonical relation

$$
\Lambda=\left\{\left(y, \eta, \iota_{\tau}(y, \eta)\right\} \subset T^{*} M \times \Sigma_{\tau} .\right.
$$

Moreover, for any s,

$$
U_{\mathbb{C}}(i \tau): W^{s}(M) \rightarrow \mathcal{O}^{s+\frac{m-1}{4}}\left(\partial M_{\tau}\right)
$$

is a continuous isomorphism.

The proof of Theorem 7.1 is barely sketched in [Bou]. However, the theorem follows almost immediately from the construction of the branched meromorphic Hadamard parametrix in Corollary 5.1, or alternatively from the analytic continuation of the Hörmander parametrix of $\$ 7.3$. It suffices to show that either is a parametrix for $U_{\mathbb{C}}(i \tau, \zeta, y)$, i.e. differs from it by an analytic kernel (smooth would be sufficient by analytic wave front set considerations). But the Hadamard parametrix construction is an exact formula and actually gives a more precise description of the singularities of $U_{\mathbb{C}}(i \tau, \zeta, y)$ than is stated in Theorem 7.1 . We briefly explain how either the Hadamard or Hörmander parametrix can be used to complete the proof.

7.1. Fourier integral distributions with complex phase. First, we review the relevant definitions (see [Ho] IV, $\S 25.5$ or [MeSj]). A Fourier integral distribution with complex phase on a manifold $X$ is a distribution that can locally be represented by an oscillatory integral

$$
A(x)=\int_{\mathbb{R}^{N}} e^{i \varphi(x, \theta)} a(x, \theta) d \theta
$$

where $a(x, \theta) \in S^{m}(X \times V)$ is a symbol of order $m$ in a cone $V \subset \mathbb{R}^{N}$ and where the phase $\varphi$ is a positive regular phase function, i.e. it satisfies

- $\operatorname{Im} \varphi \geq 0$

- $d \frac{\partial \varphi}{\partial \theta_{1}}, \ldots, d \frac{\partial \varphi}{\partial \theta_{N}}$ are linearly independent complex vectors on

$$
C_{\varphi \mathbb{R}}=\left\{(x, \theta): d_{\theta}(x, \theta)=0\right\} .
$$

- In the analytic setting (which is assumed in this article), $\varphi$ admits an analytic continuation $\varphi_{\mathbb{C}}$ to an open cone in $X_{\mathbb{C}} \times V_{\mathbb{C}}$.

Define

$$
C_{\varphi_{\mathbb{C}}}=\left\{(x, \theta) \in X_{\mathbb{C}} \times V_{\mathbb{C}}: \nabla_{\theta} \varphi_{\mathbb{C}}(x, \theta)=0\right\} .
$$

Then $C_{\varphi_{\mathbb{C}}}$ is a manifold near the real domain. One defines the Lagrangian submanifold $\Lambda_{\varphi_{\mathbb{C}}} \subset T^{*} X_{\mathbb{C}}$ as the image

$$
(x, \theta) \in C_{\varphi_{\mathbb{C}}} \rightarrow\left(x, \nabla_{x} \varphi_{\mathbb{C}}(x, \theta)\right) .
$$


7.2. Analytic continuation of the Hadamard parametrix. As in $\$ 5.1$ and $\$ 5.2$, we can express $U_{\mathbb{C}}(i \tau, \zeta, y)$ as a local Fourier integral distribution with complex phase by rewriting the Hadamard series in Corollary 5.1 as oscillatory integrals. Here we assume that $\tau>0, t \geq$ 0 .

A complication is that we can only use the complexified phase $\Gamma=t^{2}-r^{2}$ in regions of complexified $\mathbb{R} \times M \times M$ where its imaginary part is $\geq 0$. As in $\$ 5.2$, we could also use the phase $t-r$ (resp. $t+r$ ) in regions where $t+r \neq 0$ (resp. $t-r \neq 0$ ) and where the contour $\mathbb{R}_{+}$can be deformed back to itself after the the change of variables $\theta \rightarrow(t+r) \theta$.

7.3. Analytic continuation of the Hörmander parametrix. As was the case in $\mathbb{R}^{n}$ (26), the parametrix (48) admits an analytic continuation in time to a strip $\{t+i \tau: \tau<$ $\left.\tau_{a n},|t|<1\right\}$. In the space variables, the parametrix then admits an analytic continuation to complex $x, y$ satisfying $\left|r_{\mathbb{C}}(x, y)\right| \leq \tau$.

The analytically continued parametrix (53) approximates the true analytically continued Poisson kernel up to a holomorphic kernel. More preicsely, for any $x_{0} \in M$ and $\tau>0$, there exists $\varepsilon, \rho>0$ and an open neighborhood $W$ of $x_{0}$ in $M_{\tau}$ such that for $|t|<1$ and $(x, y) \in W \times W$

$$
U(t+i \tau, x, y)=\int_{T_{y}^{*} M} e^{-\tau|\xi|_{g_{y}}} e^{i\left\langle\xi, \exp _{y}^{-1}(x)\right\rangle} \mathcal{A}(t+i \tau, x, y, \xi) d \xi+R(t, x, y),
$$

where $R(t, x, y)$ is holomorphic for small $|t|$ and for $(x, y)$ near the diagonal.

The parametrix is only defined near the diagonal where $\exp _{y}^{-1}$ is defined. However one can extend it to a global holomorphic kernel away from $\mathcal{C}_{\mathbb{C}}$ by cutting off the first term of (53) with a smooth cutoff $\chi(x, y)$ supported near the diagonal in $M_{\tau} \times M_{\tau}$ and then solving a $\bar{\partial}$ problem on the Grauert tube (or a $\bar{\partial}_{b}$ problem on its boundary) to extend the kernel to be globally holmorphic (resp. CR). We refer to [Z1] for a more detailed discussion. This gives an alternative to the Hadamard parametrix construction of Corollary [5.1.

This concludes the sketch of proof of Theorem 7.1 .

\section{Tempered spectral Projector and Poisson SEMi-group as COMPlex FOURIER INTEGRAL OPERATORS}

To study the tempered spectral projection kernels (2), we further need to continue $U_{\mathbb{C}}(t, \zeta, y)$ anti-holomorphically in the $y$ variable. The discussion is similar to the holomorphic case except that we need to double the Grauert tube radius to obtain convergence. We thus have,

$$
\begin{aligned}
U_{\mathbb{C}}(t+2 i \tau, \zeta, \bar{\zeta}) & =\sum_{j} e^{(-2 \tau+i t) \lambda_{j}}\left|\varphi_{j}^{\mathbb{C}}(\zeta)\right|^{2} \\
& =\int_{\mathbb{R}} e^{i t \lambda} d_{\lambda} P_{[0, \lambda]}^{\tau}(\zeta, \bar{\zeta}) .
\end{aligned}
$$

Properties of these kernels may be obtained from kernels which are analytically continued in one variable only from the formula,

$$
\begin{aligned}
U_{\mathbb{C}}\left(t+2 i \tau, \zeta, \bar{\zeta}^{\prime}\right)= & \int_{M} U(t+i \tau, \zeta, y) U_{\mathbb{C}}\left(i \tau, y, \bar{\zeta}^{\prime}\right) d V_{g}(x) \\
= & \sum_{j} e^{(-2 \tau+i t) \lambda_{j}} \varphi_{j}^{\mathbb{C}}(\zeta) \overline{\varphi_{j}^{\mathbb{C}}\left(\zeta^{\prime}\right)} .
\end{aligned}
$$

We have, 
Proposition 8.1. For small $t, \tau>0$ and for sufficiently small $\tau \geq \sqrt{\rho}(\zeta)>0$, there exists a realization $\mathcal{B}(t, \zeta, \bar{\zeta}, \theta)$ of a formal analytic symbol $B(t, \zeta, \bar{\zeta}, \theta)$ so that as tempered distributions on $\mathbb{R} \times M_{\tau}$,

$$
U_{\mathbb{C}}(t+2 i \tau, \zeta, \bar{\zeta})=\int_{0}^{\infty} e^{i \theta((t+2 i \tau)-2 i \sqrt{\rho}(\zeta))} \mathcal{B}(t, \zeta, \bar{\zeta}, \theta) d \theta+R(t+2 i \tau, \zeta, \bar{\zeta}),
$$

where $R(t+2 i \tau, \zeta, \bar{\zeta})$ is the restriction to the anti-diagonal of a holomorphic kernel. Moreover

- $\theta((t+2 i \tau)-2 i \sqrt{\rho}(\zeta))$ is a phase of positive type.

- If $\sqrt{\rho}(\zeta)<\tau$ the entire kernel is locally holomorphic.

- If $\sqrt{\rho}(\zeta)=\tau$ then

$$
U_{\mathbb{C}}(t+2 i \tau, \zeta, \bar{\zeta})=\int_{0}^{\infty} e^{i \theta t} \mathcal{B}(t, \zeta, \bar{\zeta}, \theta) d \theta+R(t+2 i \tau, \zeta, \bar{\zeta})
$$

Proof. We use the Hadamard parametrix (Corollary 5.1) for $U(t+2 i \tau, \zeta, \bar{\zeta})$ and use (8) to simplify the phase, i.e. we write

$$
\Gamma(t+2 i \tau, \zeta, \bar{\zeta})=(t+2 i \tau-2 i \sqrt{\rho})(t+2 i \tau+2 i \sqrt{\rho})
$$

in the Hadamard parametrix in Corollary [5.1. The factors of $(t+2 i \tau+2 i \sqrt{\rho})$ are nonzero when $\tau>0$ and can be absorbed into the Hadamard coefficients. We denote the new amplitude by $\mathcal{B}$ to distinguish it from the amplitude in Corollary 5.1. We then express each term as a Fourier integral distribution of complex type with phase $t+2 i \tau-2 i \sqrt{\rho}$. It is manifestly of positive type. On $\partial M_{\tau}, t+2 i \tau-2 i \sqrt{\rho}$ simplifies to $t$.

8.1. Complexified wave group and Szegö kernels. As in [Z3] it will also be necessary for us to understand the composition $U_{\mathbb{C}}(i \tau)^{*} U_{\mathbb{C}}(i \tau)$. In this regard, it is useful to introduce the Szegö kernels $\Pi_{\tau}$ of $M_{\tau}$, i.e. the orthogonal projections

$$
\Pi_{\tau}: L^{2}\left(\partial M_{\tau}, d \mu_{\tau}\right) \rightarrow H^{2}\left(\partial M_{\tau}, d \mu_{\tau}\right),
$$

where $d \mu_{\tau}$ is the natural volume form (49). Here as above, $H^{2}\left(\partial M_{\tau}, d \mu_{\tau}\right)$ is the Hardy space of boundary values of holomorphic functions in $M_{\tau}$ which belong to $L^{2}\left(\partial M_{\tau}, d \mu_{\tau}\right)$. It is simple to prove that the restrictions of $\left\{\varphi_{\lambda_{j}}^{\mathbb{C}}\right\}$ to $\partial M_{\tau}$ is a basis of $H^{2}\left(\partial M_{\tau}, d \mu_{\tau}\right)$. The Szegö projector $\Pi_{\tau}$ is a complex Fourier integral operator with a positive complex canonical relation. The real points of its canonical relation form the graph $\Delta_{\Sigma}$ of the identity map on the symplectic cone $\Sigma_{\tau} \subset T^{*} \partial M_{\tau}$ (51). We refer to [Z3] for further background. We only need the first statement in the following:

Lemma 8.2. Let $\Psi^{s}(X)$ denote the class of pseudo-differential operators of order $s$ on $X$. Then,

- $U_{\mathbb{C}}(i \tau)^{*} U_{\mathbb{C}}(i \tau) \in \Psi^{-\frac{m-1}{2}}(M)$ with principal symbol $|\xi|_{g}^{-\left(\frac{m-1}{2}\right)}$.

- $U_{\mathbb{C}}(i \tau) \circ U_{\mathbb{C}}(i \tau)^{*}=\Pi_{\tau} A_{\tau} \Pi_{\tau}$ where $A_{\tau} \in \Psi^{\frac{m-1}{2}}\left(\partial M_{\tau}\right)$ has principal symbol $|\sigma|_{g}^{\left(\frac{m-1}{2}\right)}$ as a function on $\Sigma_{\tau}$. 
Proof. This follows from Proposition 7.1. The first statement is a special case of the following Lemma from [Z3] (Lemma 3.1): Let $a \in S^{0}\left(T^{*} M-0\right)$. Then for all $0<\tau<\tau_{\max }(g)$, we have:

$$
U(i \tau)^{*} \Pi_{\tau} a \Pi_{\tau} U(i \tau) \in \Psi^{-\frac{m-1}{2}}(M),
$$

with principal symbol equal to $a(x, \xi)|\xi|_{g}^{-\left(\frac{m-1}{2}\right)}$.

The second statement follows from Theorem 7.1 and the composition theorem for complex Fourier integral operators. We do not use it in this article and refer to [Z1] for the proof. We note that

$$
U_{\mathbb{C}}(i \tau) \circ U_{\mathbb{C}}(i \tau)^{*}\left(\zeta, \zeta^{\prime}\right)=\sum_{j} e^{-2 \tau \lambda_{j}} \varphi_{\lambda_{j}}^{\mathbb{C}}(\zeta) \overline{\varphi_{\lambda_{j}}^{\mathbb{C}}\left(\zeta^{\prime}\right)}
$$

\section{ONE TERM LOCAL WEYL LAW}

In this section, we prove Theorem 2 (1). To prove the local Weyl law we employ parametrices for the Poisson-wave kernel adapted to $e^{i(t+i \tau) \sqrt{\Delta}}$ for $\tau>0$ which are best adapted to the complex geometry.

\subsection{Proof of the local Weyl law.}

Proof. As in the real domain, we obtain asymptotics of $P_{[0, \lambda]}^{\tau}(\zeta, \bar{\zeta})$ by the Fourier-Tauberian method of relating their asymptotics to the singularities in the real time $t$ of the Fourier transform (54). We refer to [SV] (see also the Appendix of [Z1]) for background on Tauberian theorems. We follow the classical argument of [DG], Proposition 2.1, to obtain the local Weyl law with remainder one degree below the main term.

The proof is based on the oscillatory integral representation of Proposition 8.1. We are working in the case where $\sqrt{\rho}(\zeta)=\tau$ and hence can simplify it to (57).

We then introduce a cutoff function $\psi \in \mathcal{S}(\mathbb{R})$ with $\hat{\psi} \in C_{0}^{\infty}$ supported in sufficiently small neighborhood of 0 so that no other singularities of $U_{\mathbb{C}}(t+2 i \tau, \zeta, \bar{\zeta})$ lie in its support. We also assume $\hat{\psi} \equiv 1$ in a smaller neighborhood of 0 . We then change variables $\theta \rightarrow \lambda \theta$ and apply the complex stationary phase to the integral,

$$
\left.\int_{\mathbb{R}} \hat{\psi}(t) e^{-i \lambda t} U_{\mathbb{C}}(t+2 i \tau, \zeta, \bar{\zeta}) d t=\int_{\mathbb{R}} \int_{0}^{\infty} \hat{\psi}(t) e^{-i \lambda t} e^{i \theta t}(\mathcal{B}(t, \zeta, \bar{\zeta}, \theta) d \theta+R(t+2 i \tau, \zeta, \bar{\zeta}))\right) d t
$$

The second $R$ term can be dropped since it is of order $\lambda^{-M}$ for all $M>0$. In the first we change variables $\theta \rightarrow \lambda \theta$ to obtain a semi-classical Fourier integral distribution of real type with phase $e^{i \lambda t(\theta-1)}$. The critical set consists of $\theta=1, t=0$. The phase is clearly non-degenerate with Hessian determinant one and inverse Hessian operator $D_{\theta, t}^{2}$. Taking into account the factor of $\lambda^{-1}$ from the change of variables, the stationary phase expansion gievs

$$
\sum_{j} \psi\left(\lambda-\lambda_{j}\right) e^{-2 \tau \lambda_{j}}\left|\varphi_{j}^{\mathbb{C}}(\zeta)\right|^{2} \sim \sum_{k=0}^{\infty} \lambda^{\frac{n-1}{2}-k} \omega_{k}(\tau ; \zeta)
$$

where the coefficients $\left.\omega_{k}(\tau,] \zeta\right)$ are smooth for $\zeta \in \partial M_{\tau}$. However the coefficients are not uniform as $\tau \rightarrow 0^{+}$due to the factors of $(t+2 i \tau+2 i \sqrt{\rho}(\zeta))$ which were left in the denominators of the modified Hadamard parametrix. Since $t=0$ at the stationary phase point, the 
resulting expansion is equivalent to one with the large parameter $\tau \lambda$ (or $\sqrt{\rho}(\zeta) \lambda)$. The uniform expansion is then

$$
\sum_{j} \psi\left(\lambda-\lambda_{j}\right) e^{-2 \tau \lambda_{j}}\left|\varphi_{j}^{\mathbb{C}}(\zeta)\right|^{2} \sim \sum_{k=0}^{\infty}\left(\frac{\lambda}{\tau}\right)^{\frac{n-1}{2}-k} \omega_{k}(\zeta, \bar{\zeta}),
$$

where $\omega_{j}$ are smooth in $\zeta$, and $\omega_{0}=1$. The remainder has the same form.

To complete the proof, we apply the Fourier Tauberian theorem (see the Appendix ([SV]): Let $N \in F_{+}$and let $\psi \in \mathcal{S}(\mathbb{R})$ satisfy the conditions: $\psi$ is even, $\psi(\lambda)>0$ for all $\lambda \in \mathbb{R}$, $\hat{\psi} \in C_{0}^{\infty}$, and $\hat{\psi}(0)=1$. Then,

$$
\psi * d N(\lambda) \leq A \lambda^{\nu} \Longrightarrow|N(\lambda)-N * \psi(\lambda)| \leq C A \lambda^{\nu},
$$

where $C$ is independent of $A, \lambda$. We apply it twice, first in the region $\sqrt{\rho}(\zeta) \geq C \lambda^{-1}$ and second in the complementary region.

In the first region, we let $N_{\tau, \zeta}(\lambda)=P_{\tau, \lambda}(\zeta, \bar{\zeta})$. It is clear that for $\sqrt{\rho}=\tau, N_{\tau, \zeta}(\lambda)$ is a monotone non-decreasing function of $\lambda$ of polynomial growth which vanishes for $\lambda \leq 0$. For $\psi \in \mathcal{S}$ positive, even and with $\hat{\psi} \in C_{0}^{\infty}(\mathbb{R})$ and $\hat{\psi}(0)=1$, we have by (62) that

$$
\psi * d N_{\tau, \zeta}(\lambda) \leq C\left(\frac{\lambda}{\tau}\right)^{\frac{n-1}{2}},
$$

where $C$ is independent of $\zeta, \lambda$. It follows by the Fourier Tauberian theorem that

$$
N_{\tau, \zeta}(\lambda)=N_{\tau, \zeta}(\lambda) * \psi(\lambda)+O\left(\frac{\lambda}{\tau}\right)^{\frac{n-1}{2}} .
$$

Further, by integrating (62) from 0 to $\lambda$ we have

$$
N_{\tau, \zeta}(\lambda) * \psi(\lambda)=\left(\frac{\lambda}{\tau}\right)^{\frac{n-1}{2}}\left(\frac{\lambda}{\frac{n-1}{2}+1}+O(1)\right),
$$

proving (1).

To obtain uniform asymptotics in $\tau$ down to $\tau=0$, we use instead the analytic continuation of the Hörmander parametrix (53). We choose local coordinates near $x$ and write $\exp _{x}^{-1}(y)=$ $\Psi(x, y)$ in these local coordinates for $y$ near $x$, and write the integral $T_{y}^{*} M$ as an integral over $\mathbb{R}^{m}$ in these coordinates. The holomorphic extension of the parametrix to the Grauert tube $|\zeta|<\tau$ at time $t+2 i \tau$ has the form

$$
U_{\mathbb{C}}(t+2 i \tau, \zeta, \bar{\zeta})=\int_{\mathbb{R}^{n}} e^{(i t-2 \tau)|\xi|_{g_{y}}} e^{i\langle\xi, \Psi(\zeta, \bar{\zeta})\rangle} A(t, \zeta, \bar{\zeta}, \xi) d \xi
$$

Again, we use a cutoff function $\psi \in \mathcal{S}(\mathbb{R})$ with $\hat{\psi} \in C_{0}^{\infty}$ supported in sufficiently small neighborhood of 0 so that no other singularities of $E(t+2 i \tau, \zeta, \bar{\zeta})$ lie in its support and so that $\hat{\psi} \equiv 1$ in a smaller neighborhood of 0 . We write the integral in polar coordinates and obtain

$$
\begin{aligned}
& \int_{\mathbb{R}} \hat{\psi}(t) e^{-i \lambda t} U_{\mathbb{C}}(t+2 i \tau, \zeta, \bar{\zeta}) d t \\
& =\lambda^{m} \int_{0}^{\infty} \int_{\mathbb{R}} \hat{\psi}(t) e^{-i \lambda t} \int_{S^{n-1}} e^{(i t-2 \tau) \lambda r} e^{i r \lambda\langle\omega, \Psi(\zeta, \bar{\zeta})\rangle} A(t, \zeta, \bar{\zeta}, \lambda r \omega) r^{n-1} d r d \omega
\end{aligned}
$$


We then apply complex stationary phase to the $d r d t$ integral, regarding

$$
\int_{S^{n-1}} e^{i r \lambda\langle\omega, \Psi(\zeta, \bar{\zeta})\rangle} A(t, \zeta, \bar{\zeta}, \lambda r \omega) r^{m-1} d \omega
$$

as the amplitude. When $\sqrt{\rho}(\zeta) \leq \frac{C}{\lambda}$ the exponent is bounded in $\lambda$ and the integral defines a symbol. Applying stationary phase again to the $d t d \theta$ integral now gives

$$
\sum_{j} \psi\left(\lambda-\lambda_{j}\right) e^{-2 \tau \lambda_{j}}\left|\varphi_{j}^{\mathbb{C}}(\zeta)\right|^{2} \sim \sum_{k=0}^{\infty} \lambda^{n-1-k} \omega_{k}(\zeta, \bar{\zeta}),
$$

where $\omega_{k}(\zeta, \bar{\zeta})$ is smooth down to the zero section.

We apply the Fourier Tauberian theorem again, but this time with the estimates

$$
\psi * d N_{\tau, \zeta}(\lambda) \leq C \lambda^{n-1},
$$

where $C$ is independent of $\zeta$. We conclude that

$$
N_{\tau, \zeta}(\lambda)=C \lambda^{n}+O\left(\lambda^{n-1}\right)
$$

proving (2).

Corollary 9.1. For all $\zeta \in M_{\mathbb{C}}$, and with $\tau=\sqrt{\rho}(\zeta)$,

$$
c \lambda^{\frac{n+1}{2}} \leq P_{[0, \lambda]}^{\tau}(\zeta, \bar{\zeta}) \leq C \lambda^{n} .
$$

\subsection{Proof of Corollary $\underline{3}$.}

Proof. For the upper bound, we use that

$$
\sup _{\zeta \in \partial M_{\tau}}\left|\varphi_{\lambda}^{\mathbb{C}}(\zeta)\right|^{2} \leq \sup _{\zeta \in \partial M_{\tau}} \Pi_{I_{\lambda}}(\zeta, \bar{\zeta})\left|\leq \sup _{\zeta \in \partial M_{\tau}} e^{\lambda \sqrt{\rho}(\zeta)}\right| P_{I_{\lambda}}(\zeta) \mid .
$$

The upper bound stated in Corollary 3 then follows from Corollary 9.1 to Theorem 2 .

For the lower bound in (2) of Corollary 3 , we use that

$$
\left\|\varphi_{j}^{\mathbb{C}}\right\|_{L^{2}\left(\partial M_{\tau}\right)}=e^{2 \tau_{j}}\left\langle U(i \tau)^{*} U(i \tau) \varphi_{j}, \varphi_{j}\right\rangle_{L^{2}(M)} .
$$

By Lemma 8.2, the operator $U(i \tau)^{*} U(i \tau)$ is an elliptic pseudodifferential operator of order $\mu=-\frac{n-1}{2}$ (or so). Let $C>0$ be a lower bound for its symbol times $\langle\xi\rangle^{\mu}$. Then by Garding's inequality,

$$
\left\langle U(i \tau)^{*} U(i \tau) \varphi_{j}, \varphi_{j}\right\rangle_{L^{2}(M)} \geq C \lambda_{j}^{-\mu}
$$

and so

$$
\left\|\varphi_{j}^{\mathbb{C}}\right\|_{L^{2}\left(\partial M_{\tau}\right)} \geq C \lambda_{j}^{-\mu} e^{2 \tau \lambda_{j}}
$$

\section{Siciak extremal functions: Proof of Theorem 1 (1)}

In this section we prove Theorem 1. First we prove a pointwise local Weyl law in the complex domain. 
10.1. Proof of Theorem 1(2). This follows from Theorem 2 together with the following

LEMMA 10.1. [Z4 For any $\tau=\sqrt{\rho}(\zeta)>0$, and for any $\delta>0$,

$$
2 \sqrt{\rho}(\zeta)-\frac{\log |\delta|}{\lambda}+O\left(\frac{\log \lambda}{\lambda}\right) \leq \frac{1}{\lambda} \log \Pi_{[0, \lambda]}(\zeta, \bar{\zeta}) \leq 2 \sqrt{\rho}(\zeta)+O\left(\frac{\log \lambda}{\lambda}\right)
$$

hence

$$
\lim _{\lambda \rightarrow \infty} \frac{1}{\lambda} \log \Pi_{[0, \lambda]}(\zeta, \bar{\zeta})=2 \sqrt{\rho}(\zeta)
$$

Proof. For the upper bound, we use that

$$
\begin{aligned}
\Pi_{[0, \lambda]}(\zeta, \bar{\zeta}) & \leq e^{2 \lambda \sqrt{\rho(\zeta)}} \sum_{j: \lambda_{j} \in[0, \lambda]} e^{-2 \sqrt{\rho(\zeta)} \lambda_{j}}\left|\varphi_{\lambda_{j}}^{\mathbb{C}}(\zeta)\right|^{2} \\
& =e^{2 \lambda \sqrt{\rho(\zeta)}} P_{0, \lambda]}(\zeta, \bar{\zeta}) .
\end{aligned}
$$

We then take $\frac{1}{\lambda} \log$ of both sides and apply Theorem 2 to conclude the proof.

The lower bound is subtler for reasons having to do with the distribution of eigenvalues (see the Remark below). It is most natural to prove two-term Weyl asymptotics for $P_{[0, \lambda]}(\zeta, \bar{\zeta})$ and to deduce Weyl asymptotics for short spectral intervals $[\lambda, \lambda+1]$. But that requires an analysis of the singularity of the trace of the complexified wave gropup for longer times than a short interval around $t=0$ and we postpone the more refined analysis until [Z1].

Instead we use the longer intervals $[(1-\delta) \lambda, \lambda]$ for some $\delta>0$. We clearly have

$$
e^{2(1-\delta) \lambda \sqrt{\rho(\zeta)}} \sum_{j: \lambda_{j} \in[(1-\delta) \lambda, \lambda]} e^{-2 \sqrt{\rho(\zeta)} \lambda_{j}}\left|\varphi_{\lambda_{j}}^{\mathbb{C}}(\zeta)\right|^{2} \leq \Pi_{[0, \lambda]}(\zeta, \bar{\zeta})
$$

By Theorem 2 ,

$$
\begin{aligned}
\sum_{j: \lambda_{j} \in[(1-\delta) \lambda, \lambda]} e^{-2 \sqrt{\rho(\zeta)} \lambda_{j}}\left|\varphi_{\lambda_{j}}^{\mathbb{C}}(\zeta)\right|^{2} & =P_{[0, \lambda]}(\zeta, \bar{\zeta})-P_{[0,(1-\delta) \lambda]}(\zeta, \bar{\zeta}) \\
& =C_{n}(\tau)\left[1-(1-\delta)^{n}\right] \lambda^{\frac{n+1}{2}}+O\left(\lambda^{\frac{n-1}{2}}\right)
\end{aligned}
$$

Taking $\frac{1}{\lambda} \log$ then gives

$$
\frac{1}{\lambda} \log \Pi_{[0, \lambda]}(\zeta, \bar{\zeta}) \geq 2(1-\delta) \sqrt{\rho}(\zeta)-\frac{|\log \delta|}{\lambda}+O\left(\frac{\log \lambda}{\lambda}\right) .
$$

It follows that for all $\delta>0$,

$$
\liminf _{\lambda \rightarrow \infty} \frac{1}{\lambda} \log \Pi_{[0, \lambda]}(\zeta, \bar{\zeta}) \geq 2(1-\delta) \sqrt{\rho}(\zeta)
$$

The conclusion of the Lemma follows from the fact that the left side is independent of $\delta$.

Remark: The problematic issue in the lower bound is the width of $I_{\lambda}$. If $(M, g)$ is a Zoll manifold, the eigenvalues of $\sqrt{\Delta}$ form clusters of width $O\left(\lambda^{-1}\right)$ around an arithmetic progression $\left\{k+\frac{\beta}{4}\right\}$ for a certain Morse index $\beta$. Unless the intervals $I_{\lambda}$ are carefully centered around this progression, $P_{I_{\lambda}}$ could be zero. Hence we must use long spectral intervals if we do not analyze the long time behavior of the geodesic flow; for short ones no general lower bound exists. 


\subsection{Proof of Theorem 1 (1).}

Proof. We need to show that

$$
\Pi_{I_{\lambda}}^{\mathbb{C}}(\zeta, \bar{\zeta})=\sup \left\{|\varphi(\zeta)|^{2}: \varphi=\sum_{j: \lambda_{j} \in I} a_{j} \varphi_{\lambda_{j}}^{\mathbb{C}}, \quad\|a\|=1\right\} .
$$

We define the 'coherent state',

$$
\Phi_{\lambda}^{z}(w)=\frac{\Pi_{I_{\lambda}}^{\mathbb{C}}(w, \bar{z})}{\sqrt{\Pi_{I_{\lambda}}^{\mathbb{C}}(z, \bar{z})}}
$$

satisfying,

$$
\Phi_{\lambda}^{z}(w)=\sum_{j: I_{\lambda}} a_{j} \varphi_{j}^{\mathbb{C}}(w), \quad a_{j}=\frac{\overline{\varphi_{j}^{\mathbb{C}}(\zeta)}}{\sqrt{\Pi_{I_{\lambda}}^{\mathbb{C}}(z, \bar{z})}}, \quad \sum_{j}\left|a_{j}\right|^{2}=1 .
$$

Hence, $\Phi_{I_{\lambda}}^{\zeta}$ is a competitor for the sup and since $\left|\Phi_{I_{\lambda}}^{\zeta}(\zeta)\right|^{2}=\Pi_{I_{\lambda}}(\zeta, \bar{\zeta})$ one has

$$
\Pi_{I_{\lambda}}^{\mathbb{C}}(\zeta, \bar{\zeta}) \leq \sup \left\{|\psi(\zeta)|^{2}: \psi=\sum_{j: \lambda_{j} \in I} a_{j} \varphi_{j}^{\mathbb{C}}, \quad\|a\|=1\right\} .
$$

On the other hand, by the Schwartz inequality for $\ell^{2}$, for any $\psi=\sum_{j: \lambda_{j} \in I} a_{j} \varphi_{j}^{\mathbb{C}}$ one has

$$
\left|\sum_{j: \lambda_{j} \in I} a_{j} \varphi_{j}^{\mathbb{C}}\right|^{2}=|\langle a, \psi\rangle|^{2} \leq\|a\|^{2} \sum\left|\varphi_{j}^{\mathbb{C}}\right|^{2}=\Pi_{I_{\lambda}}(\zeta, \bar{\zeta})
$$

and one has

$$
\Pi_{I}^{\mathbb{C}}(\zeta, \bar{\zeta}) \geq \sup \left\{|\psi(\zeta)|^{2}: \psi=\sum_{j: \lambda_{j} \in I} a_{j} \varphi_{j}^{\mathbb{C}}, \quad\|a\|=1\right\} .
$$

Remark: Since $N\left(I_{\lambda}\right) \sim \lambda^{m-1}$,

$$
\frac{1}{\lambda} \log \Pi_{I_{\lambda}}(\zeta, \bar{\zeta})=\frac{1}{\lambda} \log \left(\sum_{j: \lambda_{j} \in I_{\lambda}}\left|\varphi_{\lambda_{j}}^{\mathbb{C}}(\zeta)\right|^{2}\right)=\max _{j: \lambda_{j} \in I_{\lambda}}\left\{\frac{1}{\lambda} \log \left|\varphi_{\lambda_{j}}^{\mathbb{C}}(\zeta)\right|^{2}\right\}+O\left(\frac{\log \lambda}{\lambda}\right) .
$$

We recall (see [Z3]) that a sequence of eigenfunctions is called ergodic if $\left\langle A \varphi_{j}, \varphi_{j}\right\rangle \rightarrow$ $\frac{1}{\mu\left(S_{g}^{*} M\right)} \int_{S_{g}^{*} M} \sigma_{A} d \mu$. The complexified eigenfunctions then satisfy $\frac{1}{\lambda_{j}} \log \left|\varphi_{j}(\zeta)\right| \rightarrow \sqrt{\rho}(\zeta)$. It follows that ergodic eigenfunctions are asymptotically maximal, i.e. have the same logarithmic asymptotics as $\Phi_{M}^{\lambda}$.

10.3. Remarks on more general extremal PSH functions. We can define a more general Siciak extremal function of a subset $E \subset M_{\tau}$ by,

$$
\Phi_{E}^{\lambda}(z)=\sup \left\{|\psi(z)|^{1 / \lambda}: \psi \in \mathcal{H}_{\lambda} ;\|\psi\|_{E} \leq 1\right\},
$$

and

$$
\Phi_{E}(z)=\sup _{\lambda} \Phi_{E}^{\lambda}(z) .
$$

It would be interesting to determine this function and the associated equilibrium measure of $E$, i.e. Monge-Ampère mass of $V_{E}^{*}$. 
This is of interest even when $E \subset M$ (i.e. is totally real). Suppose that instead of orthonormalizing the eigenfunctions $\varphi_{j}$ on $M$, we orthonormalize them on a ball $B \subset M$. Let $\left\{\varphi_{\lambda_{j}}^{B}(x)\right\}$ be the resulting orthonormal basis. We have simply changed the inner product to $\int_{B} f_{1} f_{2} d V_{g}$. We then obtain a spectral projections kernel

$$
\Pi_{[0, \lambda}^{B}(x, y):=\sum_{j: \lambda_{j} \leq \lambda} \varphi_{\lambda_{j}}^{B}(x) \varphi_{\lambda_{j}}^{B}(y) .
$$

The growth of $\Pi_{[0, \lambda]}^{B}(\zeta, \bar{\zeta})$ determines doubling estimates for eigenfunctions. Its exponential growth rate should be that of the associated pluri-complex Green's function $\log \Phi_{B}(z)=$ $\lim _{\lambda \rightarrow \infty} \frac{1}{\lambda} \log \Pi_{[0, \lambda}^{B}(\zeta, \bar{\zeta})$. It would be interesting to determine this analogue of $\sqrt{\rho}$. Its MongeAmpère mass should concentrate on $B$, so should be the metric delta-function on $B$.

\section{Analytic Continuation of eigenfunctions}

In this section, we briefly review some results about analytic continuations of eigenfunctions to Grauert tubes and then prove Proposition 1, A more detailed analysis will appear in [Z1, Z5].

A function $f$ on a real analytic manifold $M$ is real analytic, $f \in C^{\omega}(M)$, if and only if it satisfies the Cauchy estimates

$$
\left|D^{\alpha} f(x)\right| \leq K L^{|\alpha|} \alpha !
$$

for some $K, L>0$. In place of all derivatives it is sufficient to use powers of $\Delta$. In the language of Baouendi-Goulaouic [BG, BG2, BG3], the Laplacian of a compact real analytic Riemannian manifold has the property of iterates, i.e. the real analytic functions are precisely the functions satisfying Cauchy estimates relative to $\Delta$,

$$
C^{\omega}(M)=\left\{u \in C^{\infty}(M): \exists L>0, \forall k \in \mathbf{N},\left\|\Delta^{k} u\right\|_{L^{2}(M)} \leq L^{k+1}(2 k) !\right\} .
$$

It is classical that all of the eigenfunctions extend holomorphic to a fixed Grauert tube.

THEOREM 11.1. (Morrey-Nirenberg Theorem) Let $P(x, D)$ be an elliptic differential operator in $\Omega$ with coefficients which are analytic in $\Omega$. If $u \in \mathcal{D}^{\prime}(\Omega)$ and $P(x, D) u=f$ with $f \in$ $C^{\omega}(\Omega)$, then $u \in C^{\omega}(\Omega)$.

The proof shows that the radius of convergence of the solution is determined by the radius of convergence of the coefficients.

In Theorem 2 of [BG2] and Theorem 1.2 of [BGH] it is proved that the operator $\Delta$ has the iterate property if and only if, for all $b>1$, each eigenfunction extends holomorphically to some Grauert tube $M_{\tau}$ and satisfies

$$
\sup _{z \in M_{\tau}}\left|\varphi_{\lambda_{j}}^{\mathbb{C}}(z)\right| \leq b^{\lambda_{j}} \sup _{x \in M}\left|\varphi_{\lambda_{j}}(x)\right|
$$

The concept of Grauert was not actually used in these articles, so the relation between the growth rate and the Grauert tube function was not stated. But it again shows that all eigenfunctions extend to some fixed Grauert tube. 
11.1. Maximal holomorphic extension. The question then arises if all eigenfunctions extend to the maximal Grauert tube allowed by the geometry as in Definition 1.1. We conjectured in the introduction that this does hold, and now explain how it should follow from known theorems on extensions of holomorphic solutions of holomorphic PDE across non-characteristic hypersurfaces.

TheOREM 11.2. [Zer, Ho3, BSh] Let $f$ be analytic in the open set $Z \subset \mathbb{C}^{n}$ and suppose that $P(x, D) u=f$ in the open set $Z_{0} \subset Z$. If $z_{0} \in Z \cap \partial Z_{0}$ and if $Z_{0}$ has a $C^{1}$ non-characteristic boundary at $z_{0}$, then $u$ can be analytically continued as a solution of $P(x, D) u=f$ in $a$ neighborhood of $z_{0}$.

The idea of the proof is to rewrite the equation as a Cauchy problem with respect to the non-characteristic hypersurface and to apply the Cauchy Kowaleskaya theorem. To employ the theorem we need to verify that the hypsurfaces $\partial M_{\tau}$ are non-characteristic for the complexified Laplacian $\Delta_{\mathbb{C}}$, i.e. that $\sum_{i, j} g^{i j}(\zeta) \frac{\partial \sqrt{\rho}}{\partial \zeta_{i}} \frac{\partial \sqrt{\rho}}{\partial \zeta_{j}} \neq 0$. To prove this, we observe that in the real domain $g\left(\nabla r^{2}, \nabla r^{2}\right)=4 r^{2}$, an identity that was used in (13). In this formula $r^{2}=r^{2}(x, y)$ and we differentiate in $x$. We now analytically continue the identity in $x \rightarrow \zeta, y, \rightarrow \bar{\zeta}$ and differentiate only with the holomorphic derivatives $\frac{\partial}{\partial \zeta_{j}}$. From (8)), we get

$$
g_{\mathbb{C}}\left(\partial r_{\mathbb{C}}^{2}(\zeta, \bar{\zeta}), \partial r_{\mathbb{C}}^{2}(\zeta, \bar{\zeta})\right)=-4 r_{\mathbb{C}}^{2}(\zeta, \bar{\zeta})=\rho(\zeta, \bar{\zeta})>0
$$

Hence the Theorem applies and we can analytically continue eigenfunctions across any point of any $\partial M_{\tau}$ for $\tau<\tau_{g}$, the maximal radius of a Grauert tube in which the coefficients of $\Delta_{\mathbb{C}}$ are defined and holomorphic. We can take the union of the open sets where $\varphi_{j}^{\mathbb{C}}$ has a holmomorphic extension to obtain a maximal domain of holomorphy. If it fails to be $M_{\tau_{g}}$ there exists a point $\zeta$ with $\sqrt{\rho}(\zeta)<\tau_{g}$ so that $\varphi_{j}^{\mathbb{C}}$ cannot be holomorphically extended across $\partial M_{\tau}$ at $\zeta$. This contradicts the Theorem above and shows that the maximal domain must be $M_{\tau_{g}}$.

11.2. Triple inner products of eigenfunctions: Proof of Proposition 1. We start with the identity,

$$
\int_{M} \varphi_{\lambda_{j}} \varphi_{\lambda_{k}}^{2} d V_{g}=e^{-\tau \lambda_{j}}\left\langle e^{\tau \sqrt{\Delta}} \varphi_{\lambda_{j}}, \varphi_{\lambda_{k}}^{2}\right\rangle
$$

and then choose the largest value of $\tau$ for which $e^{\tau \sqrt{\Delta}} \varphi_{\lambda_{j}}, e^{\tau \sqrt{\Delta}} \varphi_{\lambda_{k}}^{2} \in W^{s}(M)$ for some $s \in \mathbb{R}$. Since

$$
\left\langle e^{\tau \sqrt{\Delta}} \varphi_{\lambda_{j}}, \varphi_{\lambda_{k}}^{2}\right\rangle=\left\langle\varphi_{\lambda_{j}}, e^{\tau \sqrt{\Delta}} \varphi_{\lambda_{k}}^{2}\right\rangle
$$

the assumption that $e^{\tau \sqrt{\Delta}} \varphi_{\lambda_{k}}^{2} \in W^{s}(M)$ implies that

$$
\int_{M} \varphi_{\lambda_{j}} \varphi_{\lambda_{k}}^{2} d V_{g} \leq C e^{-\tau \lambda_{j}}\left\|\varphi_{\lambda_{j}}\right\|_{W^{-s}} \leq\left\|e^{\tau \sqrt{\Delta}} \varphi_{\lambda_{k}}^{2}\right\|_{W^{s}} \lambda_{j}^{s} e^{-\tau \lambda_{j}} .
$$

To complete the proof it suffices to show that $e^{\tau \sqrt{\Delta}} \varphi_{\lambda_{j}} \in W^{s}(M)$ and $e^{\tau \sqrt{\Delta}} \varphi_{\lambda_{k}}^{2} \in W^{s}(M)$ for some $s \in \mathbb{R}$ as long as $\tau<\tau_{a n}(g)$. This is obvious for all $\tau$ for $\varphi_{\lambda_{j}}$ since $e^{\tau \sqrt{\Delta}} \varphi_{\lambda_{j}}=e^{\tau \lambda_{j}} \varphi_{\lambda_{j}}$. To see that it also holds for $\varphi_{\lambda_{k}}^{2}$, we note that the analytic continuation operator $\mathcal{A}(\tau)$ is given by

$$
e^{\tau \sqrt{\Delta}} f=\left(U_{\mathbb{C}}(i \tau)\right)^{-1} \mathcal{A}(\tau) f .
$$


Since $U_{\mathbb{C}}(i \tau)$ is an elliptic Fourier integral operator of finite order by Theorem 7.1 , its inverse is an elliptic Fourier integral of the opposite order. In particular, it is clear that $e^{\tau \sqrt{\Delta}} f \in$ $W^{s}(M)$ for some $s$ if and only if $\mathcal{A}(\tau) f \in \mathcal{O}^{t}\left(\partial M_{\tau}\right)$ for some $t$. In fact, $\mathcal{A}(\tau) \varphi_{\lambda_{k}}^{2}$ is real analytic on $M_{\tau}$ for any $\tau<\tau_{a n}(g)$.

To go beyond this result, one would need to know the structure of $\partial M_{\tau_{a n}(g)}$ and about the restriction of analytic continuations of eigenfunctions to it.

\section{Complex zeros of eigenfunctions: Proof of Theorem 4}

The real distribution of zeros is by definition the measure supported on the real nodal hypersurfaces $Z_{\varphi_{j}}=\left\{x \in M: \varphi_{j}(x)=0\right\}$ defined by

$$
\left\langle\left[Z_{\varphi_{j}}\right], f\right\rangle=\int_{Z_{\varphi_{j}}} f(x) d \mathcal{H}^{n-1}
$$

where $d \mathcal{H}^{n-1}$ is the $(n-1)$-dimensional Haussdorf measure induced by the Riemannian metric of $(M, g)$. The complex nodal hypersurface of an eigenfunction is defined by

$$
Z_{\varphi_{\lambda}^{\mathbb{C}}}=\left\{\zeta \in M_{\tau}: \varphi_{\lambda}^{\mathbb{C}}(\zeta)=0\right\}
$$

There exists a natural current of integration over the nodal hypersurface, given by

$$
\left\langle\left[Z_{\varphi_{\lambda}^{\mathbb{C}}}\right], \varphi\right\rangle=\frac{i}{2 \pi} \int_{M_{\tau}} \partial \bar{\partial} \log \left|\varphi_{\lambda}^{\mathbb{C}}\right|^{2} \wedge \varphi=\int_{Z_{\varphi_{\lambda}^{\mathbb{C}}}} \varphi, \quad \varphi \in \mathcal{D}^{(m-1, m-1)}\left(M_{\tau}\right) .
$$

In the second equality we used the Poincaré-Lelong formula. The notation $\mathcal{D}^{(m-1, m-1)}\left(M_{\tau}\right)$ stands for smooth test $(m-1, m-1)$-forms with support in $M_{\tau}$. The nodal hypersurface $Z_{\varphi_{\lambda}^{\mathbb{C}}}$ also carries a natural volume form $\left|Z_{\varphi_{\lambda}^{\mathbb{C}}}\right|$ as a complex hypersurface in a Kähler manifold. By

Wirtinger's formula, it equals the restriction of $\frac{\omega_{g}^{m-1}}{(m-1) !}$ to $Z_{\varphi_{\lambda}^{\mathbb{C}}}$. Hence, one can regard $Z_{\varphi_{\lambda}^{\mathbb{C}}}$ as defining the measure

$$
\left\langle\left|Z_{\varphi_{\lambda}^{\mathbb{C}}}\right|, \varphi\right\rangle=\int_{Z_{\varphi_{\lambda}^{\mathbb{C}}}^{\mathbb{C}}} \varphi \frac{\omega_{g}^{m-1}}{(m-1) !}, \quad \varphi \in C_{0}\left(M_{\tau}\right) .
$$

For background we refer to [Z3]. In that article, we proved:

THEOREM 12.1. Let $(M, g)$ be any real analytic compact Riemannian manifold with ergodic geodesic flow. Then

$$
\frac{1}{\lambda_{j_{k}}}\left[Z_{\varphi_{j_{k}}^{\mathbb{C}}}\right] \rightarrow \frac{i}{\pi} \partial \bar{\partial}|\xi|_{g}, \text { weakly in } \mathcal{D}^{\prime(1,1)}\left(B_{\varepsilon}^{*} M\right)
$$

for a full density subsequence $\left\{\varphi_{j_{k}}\right\}$.

In this section, we show that the same limit formula is valid for the entire sequence of eigenfunctions on higher rank locally symmetric manifolds studied in [AS]. 


\subsection{Plurisubharmonic functions. We put}

$$
\left\{\begin{array}{l}
\varphi_{\lambda}^{\varepsilon}=\left.\varphi_{\lambda}^{\mathbb{C}}\right|_{\partial M_{\varepsilon}} \in H^{2}\left(\partial M_{\varepsilon}\right) \\
u_{\lambda}^{\varepsilon}:=\frac{\varphi_{\lambda}^{\varepsilon}(z)}{\left\|\varphi_{\lambda}^{\varepsilon}\right\|_{L^{2}\left(\partial M_{\varepsilon}\right)}} \in H^{2}\left(\partial M_{\varepsilon}\right) \\
U_{\lambda}(z):=\frac{\varphi_{\lambda}^{\mathbb{C}}(z)}{\left\|\varphi_{\lambda}^{\varepsilon}\right\|_{L^{2}\left(\partial M_{\varepsilon}\right)}}, \quad z \in \partial M_{\varepsilon} .
\end{array}\right.
$$

Of these, $U_{\lambda}$ will play the central role. We note that $U_{\lambda}$ is CR holomorphic on $\partial M_{\tau}$. However, the normalizing factor $\left\|\varphi_{\lambda}^{\varepsilon}\right\|_{L^{2}\left(\partial M_{\varepsilon}\right)}^{-1}$ depends on $\varepsilon$, so $U_{\lambda} \notin \mathcal{O}\left(M_{\varepsilon}\right)$.

LEMMA 12.2. Let $\left\{\varphi_{j}\right\}$ be an orthonormal basis of eigenfunctions on any compact analytic Riemannian manifold $(M, g)$. Then for $\tau<\tau_{a n},\left\{\frac{1}{\lambda_{j}} \log \left|U_{j}\right|^{2}\right\}$ is pre-compact in $L^{1}\left(M_{\tau}\right)$ : every sequence has a convergent subsequence in $L^{1}\left(M_{\tau}\right)$.

Proof. As in [Z3], we use the following fact about subharmonic functions (see [Ho, Theorem 4.1.9]):

- Let $\left\{v_{j}\right\}$ be a sequence of subharmonic functions in an open set $X \subset \mathbb{R}^{m}$ which have a uniform upper bound on any compact set. Then either $v_{j} \rightarrow-\infty$ uniformly on every compact set, or else there exists a subsequence $v_{j_{k}}$ which is convergent in $L_{l o c}^{1}(X)$.

- If $v$ is subharmonic and $v_{j} \rightarrow v$ weakly in $\mathcal{D}^{\prime}\left(M_{\mathbb{C}}\right)$ then $v_{j} \rightarrow v$ in $L^{1}$.

We note that $\frac{1}{\lambda_{j}} \log \left|\varphi_{j}^{\mathbb{C}}\right|$ is plurisubharmonic and uniformly bounded above on the Grauert tube. Therefore, it either tends to $-\infty$ uniformly on compact sets of the Gruaert tube or is pre-compact in $L^{1}$. The first possibility is ruled out by the fact that it has the form $U(i \tau)^{\mathbb{C}} \varphi_{j}$ on $\partial M_{\tau}$. Hence,

$$
\left\|\varphi_{j}^{\mathbb{C}}\right\|_{L^{2}\left(\partial M_{\tau}\right)}=e^{2 \tau \lambda_{j}}\left\langle U(i \tau)^{*} U(i \tau) \varphi_{j}, \varphi_{j}\right\rangle_{L^{2}(M)} \geq e^{2 \tau \lambda_{j}} \lambda_{j}^{-\frac{m-1}{2}},
$$

by Garding's inequality (67). This contradicts the hypothesis that $\frac{1}{\lambda_{j}} \log \left|\varphi_{j}^{\mathbb{C}}\right|$ tends to zero uniformly on all compact sets, i.e. that $\left|\varphi_{j}^{\mathbb{C}}(\zeta)\right| \leq e^{-\varepsilon_{\tau} \lambda_{j}}$.

We thus have two different and independent types of weak limit problems:

- Weak limits of the $L^{2}$-normalized shell functions $U_{j}$;

- Weak limits of $\frac{1}{\lambda} \log \left|u_{j}\right|$.

LEMma 12.3. Suppose that $\left\{\varphi_{j}\right\}$ is a sequence of eigenfunctions with a unique limit measure $d \mu$ and suppose that $d \mu=\rho d \mu_{L}+\nu$ where $\rho \geq C>0$ and $\nu \perp \mu_{L}$. Then $\frac{1}{\lambda_{j}} Z_{\lambda_{j}} \rightarrow i \partial \bar{\partial}|\xi|$.

Proof. We claim that in this case $\frac{1}{\lambda_{j}} \log \left|U_{j}\right| \rightarrow 0$. Indeed, it is clear that the limsup of the left side is $\leq 0$. On the other hand, suppose that the limsup is negative on an open set $U$. Then $\int_{U}\left|U_{j}\right|^{2} \rightarrow 0$. This contradicts the assumption that limit measure has an everywhere positive Liouville component. The rest of the proof is exactly the same as in [Z3].

To complete the proof of Theorem 4, we recall (from the introduction) that Theorems 1.8, $1.9,1.10$ of $\left[\mathrm{AS}\right.$ ] prove that for co-compact lattices $\Gamma \subset S L_{n}(\mathbb{R})$, any semi-classical measure 
has a Haar component of positive weight. Hence the hypotheses of the Lemmas are satisfied by joint $\mathcal{D}$-eigenfunctions of the locally symmetric spaces.

\section{REFERENCES}

[AS] N. Anantharman and L. Silberman, A Haar component for quantum limits on locally symmetric spaces, arXiv:1009.4927.

[BG] M.S. Baouendi and C. Goulaouic, Régularité analytique et itérés d'opérateurs elliptiques dégénérés; applications. J. Functional Analysis 9 (1972), 208-248.

[BG2] M.S. Baouendi and C. Goulaouic, Itérés d'opérateurs elliptiques et prolongement de fonctions propres. Hommage au Professeur Miron Nicolescu pour son 70e anniversaire, I. Rev. Roumaine Math. Pures Appl. 18 (1973), 1495-1501.

[BG3] M.S. Baouendi and C. Goulaouic, Cauchy problem for analytic pseudo-differential operators. Comm. Partial Differential Equations 1 (1976), no. 2, 135189.

[BGH] M. S. Baouendi, C. Goulaouic, and B. Hanouzet, Caractérisation de classes de fonctions $C^{\infty}$ et analytiques sur une varit irrégulire l'aide d'un opérateur différentiel. J. Math. Pures Appl. (9) 52 (1973), 115-144.

[BT] E. Bedford and B.A. Taylor, The Dirichlet problem for a complex Monge-Ampère equation. Invent. Math. 37 (1976), no. 1, 144

[BT2] E. Bedford and B. A. Taylor, A new capacity for plurisubharmonic functions. Acta Math. 149 (1982), no. 1-2, 140.

[Be] P. Bérard, On the wave equation without conjugate points, Math. Zeit. 155 (1977), 249-276.

[BGV] N. Berline, E. Getzler and M. Vergne, Heat kernels and Dirac operators. Grundlehren Text Editions. Springer-Verlag, Berlin, 2004.

[BR] J. Bernstein and A Reznikov, Analytic continuation of representations and estimates of automorphic forms. Ann. of Math. (2) 150 (1999), no. 1, 329-352

[BL] T. Bloom and N. Levenberg, Weighted pluripotential theory in $\mathbf{C}^{N}$. Amer. J. Math. 125 (2003), no. $1,57-103$.

[BS] T. Bloom and B. Shiffman, Zeros of random polynomials on $\mathbb{C}^{m}$. Math. Res. Lett. 14 (2007), no. 3 , 469-479.

[BSh] J. M. Bony and P. Schapira, Existence et prolongement des solutions holomorphes des équations aux dérivées partielles. Invent. Math. 17 (1972), 95-105.

[Bou] L. Boutet de Monvel, Convergence dans le domaine complexe des séries de fonctions propres. C. R. Acad. Sci. Paris Sér. A-B 287 (1978), no. 13, A855-A856.

[BouG] L. Boutet de Monvel and V. Guillemin, The spectral theory of Toeplitz operators. Annals of Mathematics Studies, 99. Princeton University Press, Princeton, NJ; University of Tokyo Press, Tokyo, 1981.

[BoSj] L. Boutet de Monvel and J. Sjöstrand, Sur la singularité des noyaux de Bergman et de Szegö, Asterisque 34-35 (1976), 123-164.

[Br] H. J. Bremermann, On a generalized Dirichlet problem for plurisubharmonic functions and pseudoconvex domains. Characterization of Silov boundaries. Trans. Amer. Math. Soc. 911959246276.

[BW] F. Bruhat and H. Whitney, Quelques propriétés fondamentales des ensembles analytiques-réels. Comment. Math. Helv. 331959 132-160.

[DF] H. Donnelly and C. Fefferman, Nodal sets of eigenfunctions on Riemannian manifolds, Invent. Math. 93 (1988), 161-183.

[DG] J. J. Duistermaat and V. W. Guillemin, The spectrum of positive elliptic operators and periodic bicharacteristics. Invent. Math. 29 (1975), no. 1, 39-79.

[DH] J. J. Duistermaat and L. Hörmander, Fourier integral operators II, Acta Math. 128 (1972), no. 3-4, 183269.

[Gar] P. R. Garabedian, Partial differential equations. Reprint of the 1964 original. AMS Chelsea Publishing, Providence, RI, 1998.

[GeSh] I. M. Gelfand and G. E. Shilov, Generalized Functions, Vol. 1, Academic Press (1964). 
[GLS] F. Golse, E. Leichtnam, and M. Stenzel, Intrinsic microlocal analysis and inversion formulae for the heat equation on compact real-analytic Riemannian manifolds. Ann. Sci. École Norm. Sup. (4) 29 (1996), no. 6, 669-736.

[Gr] H. Grauert, Über Modifikationen und exzeptionelle analytische Mengen, Math. Annalen 146 (1962), $331-368$.

[GZ] V. Guedj and A. Zeriahi, Intrinsic capacities on compact Kähler manifolds. J. Geom. Anal. 15 (2005), no. 4, 607-639.

[G] V. Guillemin, Paley-Wiener on manifolds. In The Legacy of Norbert Wiener: A Centennial Symposium (Cambridge, MA, 1994), 85-91, Proc. Sympos. Pure Math., 60, Amer. Math. Soc., Providence, RI, 1997.

[GS1] V. Guillemin and M. Stenzel, Grauert tubes and the homogeneous Monge-Ampère equation. J. Differential Geom. 34 (1991), no. 2, 561-570.

[GS2] V. Guillemin and M. Stenzel, Grauert tubes and the homogeneous Monge-Ampère equation. II. J. Differential Geom. 35 (1992), no. 3, 627-641.

[H] J. Hadamard, Lectures on Cauchy's problem in linear partial differential equations. Dover Publications, New York, 1953.

[HL] Q. Han and F.-H. Lin, Nodal sets of solutions of elliptic differential equations, book in preparation (2008).

[HW] F. R. Harvey and R. O. Wells, Zero sets of non-negative strictly plurisubharmonic functions. Math. Ann. 201 (1973), 165170.

[HS] B. Helffer and J. Sjöstrand, Multiple wells in the semiclassical limit I, Comm. Partial Differential Equations 9 (1984), 337-408.

[Hel] S. Helgason, Groups and geometric analysis. Integral geometry, invariant differential operators, and spherical functions. Mathematical Surveys and Monographs, 83. American Mathematical Society, Providence, RI, 2000.

[Ho] L. Hörmander, The Analysis of Linear Partial Differential Operators, Volumes III-IV, SpringerVerlag Berlin Heidelberg, 1983.

[Ho2] L. Hörmander, The spectral function of an elliptic operator. Acta Math. 121 (1968), 193-218.

[Ho3] L. Hörmander, Linear Partial Differential Operators, vol. 116. Springer Grundlehren, Berlin (1976)

[IZ] C. Itzykson and J.B. Zuber, Quantum field theory. International Series in Pure and Applied Physics. McGraw-Hill International Book Co., New York, 1980.

rge(1-YALE)

[JL] J. Jorgenson and S. Lang, Analytic continuation and identities involving heat, Poisson, wave and Bessel kernels. (English summary) Math. Nachr. 258 (2003), 4470.

[J] M. S. Joshi, Complex powers of the wave operator, Portugaliae Math. 54 (1997), 345- 362.

[KM] S-J. Kan and D. Ma, On rigidity of Grauert tubes over Riemannian manifolds of constant curvature. Math. Z. 239 (2002), no. 2, 353-363.

[K] M. Klimek, Pluripotential Theory. London Mathematical Society Monographs. New Series, 6, Oxford University Press, New York, 1991.

[KS] B. Krötz and H. Schlichtkrull, Holomorphic extension of eigenfunctions. Math. Ann. 345 (2009), no. 4, 835841.

[Lax] P. Lax, Asymptotic solutions of oscillatory initial value problems. Duke Math. J. 241957 627-646.

[Leb] G. Lebeau, Fonctions harmoniques et spectre singulier. Ann. Sci. École Norm. Sup. (4) 13 (1980), no. 2, 269-291.

[LS1] L. Lempert and R. Szöke, Global solutions of the homogeneous complex Monge-Ampère equation and complex structures on the tangent bundle of Riemannian manifolds. Math. Ann. 290 (1991), no. 4, 689-712.

[Lin] F.H. Lin, Nodal sets of solutions of elliptic and parabolic equations. Comm. Pure Appl. Math. 44 (1991), no. 3, 287-308.

[Mar] A. Martinez, Estimates on complex interactions in phase space, Math. Nachr. 167 (1994), 203-254. 
[MeSj] A. Melin and J. Sjöstrand, Fourier integral operators with complex-valued phase functions.Fourier integral operators and partial differential equations (Colloq. Internat., Univ. Nice, Nice, 1974), pp. 120223. Lecture Notes in Math., Vol. 459, Springer, Berlin, 1975.

[Miz] S. Mizohata, Analyticity of the fundamental solutions of hyperbolic systems. J. Math. Kyoto Univ. 1 1961/1962 327-355.

[Miz2] S. Mizohata, Analyticity of solutions of hyperbolic systems with analytic coefficients. Comm. Pure Appl. Math. 141961 547-559.

[R] M. Riesz, L'intégrale de Riemann-Liouville et le problme de Cauchy. Acta Math. 81, (1949). 1-223.

[RZ] Y. A. Rubinstein and S. Zelditch, Complex zeros of integrable eigenfunctions, (in preparation).

[Saf] Y. Safarov, Asymptotics of a spectral function of a positive elliptic operator without a nontrapping condition. (Russian) Funktsional. Anal. i Prilozhen. 22 (1988), no. 3,53-65, 96 translation in Funct. Anal. Appl. 22 (1988), no. 3, 213-223 (1989).

[SV] Y. Safarov and D. Vassiliev, The asymptotic distribution of eigenvalues of partial differential operators. Translated from the Russian manuscript by the authors. Translations of Mathematical Monographs, 155. American Mathematical Society, Providence, RI, 1997.

[Sar] P. Sarnak, Integrals of products of eigenfunctions. Internat. Math. Res. Notices 1994, no. 6, 251 ff.,

[Sic] J. Siciak, Extremal plurisubharmonic functions in $C^{n}$. Ann. Polon. Math. 39 (1981), 175-211.

[Sic2] J. Siciak, On some extremal functions and their applications in the theory of analytic functions of several complex variables. Trans. Amer. Math. Soc. 1051962 322-357.

[Sj] J. Sjöstrand, Singularités analytiques microlocales. Astérisque, 95, 1-166, Soc. Math. France, Paris, 1982.

[St] E. M. Stein, Singular integrals and differentiability properties of functions. Princeton Mathematical Series, No. 30 Princeton University Press, Princeton, N.J. 1970

[Sz] R. Szoke, Complex structures on tangent bundles of Riemannian manifolds. Math. Ann. 291 (1991), no. 3, 409-428

[T] M.E. Taylor, Noncommutative harmonic analysis. Mathematical Surveys and Monographs, 22. American Mathematical Society, Providence, RI, 1986.

[To] J. A. Toth, Eigenfunction decay estimates in the quantum integrable case. Duke Math. J. 93 (1998), no. 2, 231-255.

[TZ] J. A. Toth and S. Zelditch, Counting nodal lines which touch the boundary of an analytic domain, Jour. Diff. Geom. 81 (2009), 649- 686 (arXiv:0710.0101).

[Za] V. P. Zaharjuta, Extremal plurisubharmonic functions, orthogonal polynomials, and the BernsteinWalsh theorem for functions of several complex variables (Russian), Ann. Polon. Math. 33 (1976/77), $137-148$.

[Z1] S. Zelditch, Pluri-potential theory on Grauert tubes of real analytic Riemannian manifolds, II (in preparation).

[Z2] S. Zelditch, Real and complex zeros of Riemannian random waves, Spectral analysis in geometry and number theory, 321-342, Contemp. Math., 484, Amer. Math. Soc., Providence, RI, 2009.

[Z3] S. Zelditch, Complex zeros of real ergodic eigenfunctions, Invent. Math. 167 (2007), no. 2, 419-443.

[Z4] S. Zelditch, Szegö kernels and a theorem of Tian, IMRN 6 (1998), 317-331.

[Z5] S. Zelditch, CBMS Lectures (in preparation).

[Zer] M. Zerner, Domain d'holomorphie des fonctions vérifiant une équation aux dérivées partielles, C. R. Acad. Sci. Paris Ser. I Math. 272 (1971) 1646-1648.

Department of Mathematics, Northwestern University, Evanston, IL 60208, USA

E-mail address: zelditch@math.northwestern.edu 